\title{
Combinando estrategias comunitarias y política gubernamental para prevenir la infección del VIH en la República Dominicana
}

\author{
Deanna Kerrigan \\ Luis Moreno \\ Santo Rosario \\ Bayardo Gomez \\ Hector Jerez
}

See next page for additional authors

Follow this and additional works at: https://knowledgecommons.popcouncil.org/departments_sbsr-hiv

Part of the Family, Life Course, and Society Commons, Gender and Sexuality Commons, International Public Health Commons, and the Medicine and Health Commons

How does access to this work benefit you? Let us know!

\section{Recommended Citation}

Kerrigan, Deanna, Luis Moreno, Santo Rosario, Bayardo Gomez, Hector Jerez, Ellen Weiss, Johannes van Dam, Eva Roca, Clare Barrington, and Michael D. Sweat. 2004. "Combinando estrategias comunitarias y política gubernamental para prevenir la infección del VIH en la República Dominicana," Horizons Informe Final. Washington, DC: Population Council. 


\section{Authors}

Deanna Kerrigan, Luis Moreno, Santo Rosario, Bayardo Gomez, Hector Jerez, Ellen Weiss, Johannes van Dam, Eva Roca, Clare Barrington, and Michael D. Sweat 


\title{
Hgrizons N
}

Combinando Estrategias Comunitarias y

Política Gubernamental para

Prevenir la Infección del VIH

\author{
en la República Dominicana
}
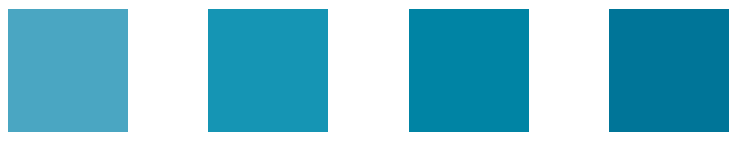

Horizons Program

Johns Hopkins University

Centro de Orientación e Investigación Integral

Centro de Promoción y Solidaridad Humana International Center for Research on Women 


\title{
Combinando Estrategias Comunitarias y Política Gubernamental para Prevenir la I nfección del VI H en la República Dominicana
}

\author{
Deanna Kerrigan ${ }^{1}$, Luis Morenoㄹ, Santo Rosario², Bayardo Gomez ${ }^{3}$, \\ Hector Jerez ${ }^{3}$, Ellen Weiss ${ }^{4}$, J ohannes van Dam ${ }^{5}$, Eva Roca ${ }^{6}$, \\ Clare Barrington ${ }^{7}$, and Michael Sweat ${ }^{7}$
}

\footnotetext{
${ }^{1}$ Horizons/J ohns Hopkins University

${ }^{2}$ Centro de Orientación e Investigación Integral (COIN)

${ }^{3}$ Centro de Promoción y Solidaridad Humana (CEPROSH)

${ }^{4}$ Horizons/International Center for Research on Women (ICRW)

${ }^{5}$ Horizons/Population Council

${ }^{6}$ ICRW, anteriormente Horizons/Population Council

${ }^{7}$ Johns Hopkins University
} 


\section{Agradecimientos}

Los autores quisieran agradecer a los participantes del estudio por su tiempo, dedicación e ideas, y a los miembros de las organizaciones locales contrapartes que ayudaron en la investigación e implementación de las actividades de intervención. Estos incluyen a Martha Butler de Lister del proyecto CONECTA de Family Health International (anteriormente de la Dirección General de Control de Infecciones de Transmisión Sexual y SIDA [DIGECITSS]); William Hernández, Rosa Flores, Juliana Martínez, y Yafmari Feliz de DIGECITSS; Alexandra Lister, Nancy Gómez, Ramón Hipólito, Nila Fernández, Carmen Hernández, Belkis Altagracia de la Rosa, Juan Familia, Elisa Polanco y Arelis Moore del Centro de Promoción y Solidaridad Humana (CEPROSH); Francisca Ferreira, Juana Clase y Melchor Moya del Centro de Orientación e Investigación Integral (COIN); Carmen Lorenzo, Marina Torres y Marina Ureña del Movimiento de Mujeres Unidas (MODEMU); Margarita Quiñones del Instituto Dermatológico y Cirugía de la Piel (IDCP); los inspectores de salud gubernamentales y sus coordinadores regionales de la Dirección de Saneamiento Ambiental de la Secretaria de Estado de Salud Pública y Asistencia Social (SESPAS); los médicos de las clínicas públicas que dan servicio a trabajadoras sexuales y sus coordinadores regionales de las Direcciones Provinciales de Salud de la SESPAS; y Tito Coleman del proyecto AccionSIDA de la Academy for Educational Development (AED).

También, quisiéramos agradecer a las siguientes personas quienes ayudaron a revisar y refinar este informe, entre ellos Carol Jenkins, consultora independiente y la anteriormente consejera regional de VIH para el oriente cercana con el Buró de Asia del USAID; Jerry Jennings de USAID; Anneke van den Hoek, director del Servicio de Salud Municipal de Ámsterdam en Holanda; y Naomi Rutenberg, Lewis Ndhlovu, y Eka EsuWilliams del programa Horizons. Finalmente, gracias a Andy Fisher, director del programa Horizons/Population Council; Barbara de Zalduondo de Social and Scientific Systems (anteriormente de USAID); y David Stanton de USAID por su motivación y apoyo durante el proceso de la investigación.

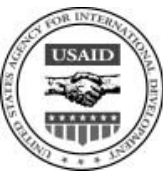

Este estudio fue apoyado por el programa Horizons, el cual es implementado por Population Council en colaboración con International Center for Research on Women, International HIV/AIDS Alliance, Program for Appropriate Technology in Health, Tulane University, Family Health International, y Johns Hopkins University. Horizons es financiado por el U.S. Agency for International Development (USAID), bajo los términos del HRN-A-00-97-00012-00. Las opiniones expresadas adentro son de los autores y no necesariamente reflejan las opiniones de USAID.

Publicado en Marzo 2004.

\section{(2) Population Council}

Population Council es una institución no gubernamental, internacional, sin fines de lucro, que busca mejorar el bienestar y salud reproductiva de las generaciones actuales y futuras en el mundo y ayudar a lograr un equilibrio humano, de equidad y sostenible entre personas y recursos. Population Council conduce investigación biomédica, de ciencia social y de salud pública y ayuda a crear capacidades para investigación en países en desarrollo. Establecido en 1952, el Council es gobernado por un una directiva internacional. Su sede en Nueva York apoya una red global de oficinas regionales y de países.

Copyright $\odot 2004$ The Population Council Inc.

Este documento puede ser reproducido entero o en parte sin el permiso de Population Council siempre y cuando se de una cita completa y la reproducción no sea para propósitos comerciales.

Cita sugerida: Kerrigan, Deanna, Luis Moreno, Santo Rosario, Bayardo Gomez, Hector Jerez, Ellen Weiss, Johannes van Dam, Eva Roca, Clare Barrington, y Michael Sweat. 2004. "Combinando Estrategias Comunitarias y Política Gubernamental para Prevenir la infección del VIH en la Republica Dominicana," Horizons Informe Final. Washington, D.C.: Population Council. 


\section{Tabla de Contenidos}

\section{Resumen Ejecutivo}

Introducción 5

$\begin{array}{lc}\text { Programa Tailandés } 100 \text { por ciento Condón } & 6\end{array}$

Adaptando el Programa Tailandés a la RD 6

Métodos 8

$\begin{array}{lc}\text { Contexto de la investigación } & 8\end{array}$

Diseño del estudio 9

La intervención 9

Recolección de datos y procedimientos éticos $\quad 13$

Medidas 15

$\begin{array}{ll}\text { Manejo de datos y análisis } & 16\end{array}$

Resultados $\quad 18$

$\begin{array}{ll}\text { Características de la muestra } & 18\end{array}$

Cambios pre- a post-intervención en el riesgo relacionado al VIH 19

Otros resultados de la intervención $\quad 26$

$\begin{array}{ll}\text { Análisis de costo-efectividad } & 27\end{array}$

Resultados 36

Discusión $\quad 43$

Limitaciones de la I nvestigación 46

Conclusiones 47

$\begin{array}{ll}\text { Referencias } & 48\end{array}$ 



\section{Resumen Ejecutivo}

Programas efectivos para evitar nuevas infecciones del VIH entre trabajadoras sexuales y sus parejas, y por lo tanto la población en general, son componentes críticos de estrategias nacionales de prevención del VIH. Generalmente, los esfuerzos de prevención se han concentrado en intervenciones que alcanzan al nivel individual al los miembros de estos grupos vulnerables, tales como promoción de condones y manejo de infecciones de transmisión sexual (ITS). Actualmente, muchos investigadores y gerentes de programas se encuentran dirigiéndose a intervenciones "ambientales-estructurales" que toman en cuenta los contextos físicos, sociales y políticos donde ocurre el comportamiento individual.

Recientemente dos tipos de intervenciones ambientales-estructurales han demostrado aumentos en el uso del condón y reducciones en la prevalencia de ITS en las trabajadoras sexuales. Un enfoque consiste en la movilización y desarrollo comunitario para crear un compromiso colectivo con la prevención, tal como el Proyecto Sonagachi en Calcuta, India (Jana 1998). El segundo enfoque consiste en iniciativas patrocinadas por el gobierno, incluyendo el "Programa 100\% Condones" implementado en Tailandia que utiliza una política gubernamental que requiere el uso de condones en burdeles (Rojanapithayakorn 1996).

Un estudio recientemente realizado por Horizons, en conjunto con dos organizaciones nogubernamentales (ONGs) dominicanas-Centro de Orientación e Investigación Integral (COIN) y Centro de Promoción y Solidaridad Humana (CEPROSH) — y el Programa Nacional del Control de ITS y SIDA (DIGECITSS) evaluó el impacto de dos modelos ambientales-estructurales en disminuir el riesgo de VIH en trabajadoras sexuales en la República Dominicana y comparó el costo-efectividad de los dos modelos. Los modelos, construidos sobre la base de años de experiencia adquirida a través de programas de educación entre pares de trabajadoras sexuales, combinan las fortalezas de iniciativas de solidaridad comunitaria y de políticas gubernamentales, e involucran a miembros de la comunidad en el desarrollo de ambos programa y política.

\section{Descripción de la I ntervención}

Los modelos de intervención fueron desarrollados después de una extensa investigación formativa y de consultación con líderes de pares. Los modelos fueron implementados en 68 establecimientos de sexo en dos ciudades dominicanas. En Santo Domingo, la capital del país, se implementó un modelo basado en la solidaridad comunitaria con respecto al uso $100 \%$ de condones, mientras que en Puerto Plata, una ciudad costera más pequeña ubicada en el norte del país y con altos niveles de turismo, el modelo de solidaridad comunitaria fue combinado con la política y regulación gubernamental.

Actividades para construir la solidaridad incluyeron talleres y reuniones con trabajadoras sexuales, dueños y administradores de establecimiento y otros empleados, como porteros y disc jockeys, para fortalecer el compromiso colectivo con la prevención del VIH/SIDA, y particularmente para apoyar 


\section{Hgrizons}

a las trabajadoras sexuales en el uso de condones con sus parejas. Estas reuniones también se enfocaron en explorar temas de confianza e intimidad en la negociación del uso de condones entre trabajadoras sexuales y sus parejas fijas que pagan y no pagan.

Para fortalecer el compromiso colectivo con la prevención, se le recomendó a cada dueño de establecimiento de sexo exponer afiches de $100 \%$ Condones y otros materiales para aumentar la conciencia, y mantener recipientes transparentes de vidrio llenas de condones en cada establecimiento. Otros recordatorios ambientales para apoyar el uso de condones incluyeron mensajes del disc jockey acerca de sexo seguro, mesas de información en las entradas de los establecimientos y teatro participativo con clientes masculinos.

En Puerto Plata, se utilizó el mismo modelo de intervención pero se incluyó además una política de $100 \%$ condones, patrocinada por el gobierno, que requirió el uso de condones entre trabajadoras sexuales y todos sus clientes. Los dueños fueron informados que ellos, y no las trabajadoras sexuales, eran responsables de asegurar el cumplimiento con la política y las actividades del programa. En caso de faltas de cumplimiento, el gobierno iniciaba un proceso de advertencias, multas y otras sanciones, incluyendo la clausura del establecimiento.

\section{Métodos}

Los investigadores utilizaron un diseño de evaluación pre/post-examen para evaluar los dos programas, con plazo de un año, que fueron realizados en 34 establecimientos de sexo en cada ciudad. Encuestas estructuradas y pruebas no-rutinarias de ITS fueron realizadas con una muestra transversal de aproximadamente 200 trabajadoras sexuales, de cómo mínimo 18 años, que trabajaban en los establecimientos de sexo que formaban parte del estudio. Las mujeres fueron reclutadas en clínicas de salud gubernamentales en cada ciudad, pre- y post- intervención. Los datos fueron recolectados al inicio y al final del período de intervención de 12 meses.

En ambas ciudades, la edad mediana de las trabajadoras sexuales que participaron en el estudio fue de aproximadamente 25 años; el número mediano de años de educación fueron siete. Más del 75 por ciento eran solteras, con casi dos-tercios reportando tener una pareja fija. El número mediano de salidas con clientes reportadas por las trabajadoras sexuales en la última semana fue dos en Puerto Plata (rango: 0-32) y uno en Santo Domingo (rango: 0-30).

Inspectores de salud gubernamentales acompañados por personal de las ONGs visitaron los establecimientos en ambas ciudades mensualmente para evaluar el cumplimiento con cinco elementos claves: la presencia de afiches de $100 \%$ Condones, la disponibilidad de cómo mínimo 100 condones en cada sitio, visibilidad y accesibilidad de condones para clientes y trabajadoras sexuales, la asistencia a chequeos mensuales de ITS de todas las trabajadoras sexuales del establecimiento y la falta de un diagnóstico positivo para ITS en las trabajadoras sexuales del establecimiento.

Además, personal masculino de las ONGs pasando como clientes realizaron observaciones participativas pre- y post-intervención en una muestra al azar de 64 trabajadoras sexuales en cada 
ciudad. Siguiendo un protocolo estricto de investigación, cada "cliente" seleccionó una trabajadora sexual y conversó con ella por aproximadamente 30 minutos en una mesa del establecimiento. Se le preguntó si estaba dispuesta a tener sexo sin un condón, presentándole cuatro razones, desarrolladas durante investigación formativa, por las cuales el no quería usar un condón.

Este estudio fue aprobado por los comités de ética del Population Council y la Universidad Johns Hopkins. DIGECITSS también realizó una revisión técnica y ética del estudio.

\section{Resultados Claves}

El uso consistente de condones (UCC) incrementó significativamente en cada ciudad, aunque con diferentes tipos de clientes. El UCC con clientes nuevos en el último mes incrementó significativamente en las trabajadoras sexuales en Santo Domingo, de 75 por ciento a 94 por ciento ( $\mathrm{p}<0.001$ ). En Puerto Plata, donde el UCC con clientes nuevos ya tenía una tasa alta de 96 por ciento, la tasa aumentó a 99 por ciento. Solo en Puerto Plata incrementó significativamente el UCC con parejas fijas que pagan y no pagan, de 13 por ciento a 29 por ciento $(\mathrm{p}<0.001)$; en Santo Domingo, el UCC con parejas fijas incrementó levemente, de 15 por ciento a 18 por ciento. Estas cifras son particularmente importantes dado que el uso de condones con parejas fijas que pagan y no pagan fue mucho más bajo comparado con el uso de condones con clientes nuevos, preintervención. Adicionalmente, el estudio encontró que la mayoría de actos de sexo en el último mes entre todas las participantes ocurrieron con parejas fijas que pagan, haciendo el aumento en Puerto Plata particularmente importante.

La capacidad de las trabajadoras sexuales a rechazar el sexo inseguro aumentó significativamente solamente en Puerto Plata. Resultados de las observaciones participativas en Puerto Plata demostraron que el porcentaje de trabajadoras sexuales que rechazaron el sexo sin condón después de oír las cuatro razones incrementó de 50 por ciento a 79 por ciento $(\mathrm{p}<0.001)$. En Santo Domingo, el rechazo del sexo inseguro también incrementó de 64 por ciento a 72 por ciento, pero la diferencia no fue estadísticamente significativa.

Las tasas de ITS bajaron casi 40 por ciento de pre- a post-intervención. En ambas ciudades, hubo reducciones significativas en la proporción de mujeres con resultados positivos de una o más de tres ITS (gonorrea, Tricomoniasis y/o Clamidia), con descensos un poco más sustantivos en Puerto Plata ( $\mathrm{p}<0.003)$, comparado con Santo Domingo $(\mathrm{p}<0.014)$. Estas reducciones generales se atribuyen mayormente a los descensos en ITS específicas: en Santo Domingo, la prevalencia de Clamidia bajó significativamente de 16 por ciento a 9 por ciento $(\mathrm{p}<0.029)$, mientras que en Puerto Plata, la prevalencia de Tricomoniasis bajó significativamente de 10 por ciento a 4 por ciento $(\mathrm{p}<0.024)$.

EI UCC en las trabajadoras sexuales fue asociado con niveles más altos de exposición a la intervención y el cumplimiento de su establecimiento con la intervención. En análisis multivariable, el equipo de investigación encontró que la probabilidad del UCC en las trabajadoras sexuales con todas sus parejas sexuales en el último mes fue 1.84 (IC 1.07-3.17) veces más en las que reportaron niveles altos de exposición a la intervención. El UCC también fue 2.33 (IC 1.01- 


\section{Hgrizons}

5.39) veces más en las trabajadoras sexuales de los establecimientos con el nivel más alto de cumplimiento observado con los elementos claves de la intervención.

Los establecimientos de sexo que participaron en la intervención en Puerto Plata demostraron un aumento significativo en el cumplimiento observado con elementos de la intervención. La exposición reportada a la intervención aumentó significativamente en las trabajadoras sexuales en ambas ciudades. Sin embargo, los niveles de cumplimiento observado con los cinco elementos claves de la intervención aumentaron significativamente de un promedio de 2.6 en el primer mes a 4.7 en el duodécimo mes $(\mathrm{p}<0$.001) solamente en Puerto Plata, donde se implementó una política gubernamental requiriendo el uso de condones y un sistema graduado de sanciones para los que no lo cumplían.

El modelo de Puerto Plata, que incluyó regulación gubernamental, fue más costo-efectivo que el modelo de Santo Domingo. Los investigadores hicieron un análisis de costo demostrando que mientras el costo para implementar los dos modelos es parecido, el modelo de intervención que combinó regulación y supervisión gubernamental es aproximadamente dos veces y medio más costo-efectivo. El costo por infección de VIH evitada en Puerto Plata, de US \$9,843, es sustantivamente menos que en Santo Domingo, de US \$25,574. Al convertirlo, el costo por año de vida ajustado por la discapacidad (AVAD) salvado es de US \$414 en Puerto Plata, comparado con US \$1,075 en Santo Domingo.

\section{Conclusiones}

En ambas ciudades hubo mejoramientos de pre- a post-intervención en las variables claves del estudio: el uso consistente de condones, el rechazo del sexo inseguro y la prevalencia de ITS. Sin embargo, el tipo y el nivel de estos cambios variaron con el modelo de intervención. El modelo de Puerto Plata, que incluyó una política gubernamental y regulación, parece haber sido más exitoso en estimular aumentos significativos en el UCC con parejas fijas que pagan y que no pagan.

Asimismo, la capacidad para rechazar el sexo inseguro por las trabajadoras sexuales aumentó significativamente solamente en Puerto Plata.

La triangulación de estos resultados demuestra que mientras que ambos modelos tuvieron un impacto positivo en reducir la vulnerabilidad al VIH, el impacto de la intervención parece haber sido más amplio en Puerto Plata. Los datos de costo-efectividad también muestran que el modelo de Puerto Plata es mucho más costo-efectivo que el modelo de Santo Domingo. Los resultados positivos en Puerto Plata probablemente están vinculados con la capacidad del modelo combinado de esa ciudad solidaridad comunitaria más política y regulación gubernamental para alcanzar niveles más altos de cumplimiento con los elementos claves de la intervención comparados con Santo Domingo.

Mientras que existen ciertas limitaciones del estudio (ej. diseño pre-post, solo un modelo implementado en cada ciudad), triangulación de los datos de diferentes fuentes destaca que el modelo combinado basado en la solidaridad y la política gubernamental es un paquete de intervención viable, aceptable y efectivo para la República Dominicana. Con financiamiento del 
Fondo Global, el Banco Mundial y la USAID, las ONGs implementadoras en coordinación con el gobierno dominicano están actualmente expandiendo el modelo combinado en áreas del país en donde el sexo comercial es prevalente. 


\section{Hgrizons}

\section{Introducción}

Dado sus múltiples parejas sexuales, trabajadoras sexuales, especialmente las de lugares de bajo nivel socioeconómico, son particularmente vulnerables a infectarse con VIH y otras infecciones de transmisión sexual (ITS) (Gysels et al. 2002; Ghys et al. 2001; Kalipeni 2000; Campbell 2000; Gray et al. 1997; Aklilu et al. 2001). Estudios epidemiológicos de muchos países han documentado tasas de infección de VIH significativamente más altas en trabajadoras sexuales, comparadas con otros grupos de la población dentro de la misma área geográfica y contexto cultural (Asamoah-Adu et al. 2001; Quan et al. 2000; Mehendale et al. 1995).

Por ejemplo, de acuerdo a la reciente Encuesta Demográfica y de Salud dentro de la cual se realizaron pruebas de VIH orales con hombres y mujeres de 15 a 49 años en la República Dominicana, se estima la prevalencia del VIH en la población general en 1.0 por ciento (CESDEM 2003). Sin embargo, la prevalencia del VIH nacional en trabajadoras sexuales es aproximadamente 7 por ciento, con tasas de infección llegando tan altas como 12 por ciento en algunas regiones del país (SESPAS 2000). Aunque la República Dominicana es un país relativamente pequeño con aproximadamente 8.4 millones de habitantes, el número de trabajadoras sexuales ha sido estimado conservadoramente entre 60,000 y 100,000, indicando que un número sustancial de mujeres están en alto riesgo de infectarse con VIH dado a sus múltiples parejas sexuales (AIDSCAP 1993; COIN 2000).

Trabajadoras sexuales son, entonces, un grupo meta en la implementación de programas de prevención del VIH que tratan tanto de prevenir su salud como de evitar miles de potenciales nuevas infecciones en la población general. Tales programas se han fiado con frecuencia en intervenciones en el nivel individual, como por ejemplo educación entre pares, promoción y distribución de condones y manejo de ITS. Algunos programas en países en desarrollo han demostrado incrementos en la tasa del uso de condones o reducciones en las ITS en trabajadoras sexuales utilizando una o más de estas estrategias (Laga et al. 1994; Bhave et al. 1995; Ngugi et al. 1996; Ford et al. 1996).

Sin embargo, hay nuevas perspectivas y estrategias que están informando sobre el diseño de programas en el contexto del trabajo sexual. Con el tiempo, ha emergido un consenso en la salud pública de que el cambio de comportamiento de alto riesgo y reducción de vulnerabilidad al VIH no ocurre en un contexto simple sino que es el producto de muchos procesos y esfuerzos individuales, relacionales, socio-culturales, ambientales-estructurales y tecnológicos (Sweat and Denison 1995; Tawil et al. 1995; O'Reilly and Piot 1996; Aggleton 1997; Merson et al. 2000; Parker et al. 2000; Auerbach and Coates 2000; Waldo and Coates 2000). En respuesta, programas de prevención de VIH cada vez mas están enfocando su atención en intervenciones "ambientales estructurales" en el trabajo sexual que buscan dirigirse a la amplitud de estos contextos (Sweat and Denison 1995; Sumartojo 2000). A la vez, estrategias de prevención adicionales han sido desarrolladas (UNAIDS 2002; Moreno and Kerrigan 2000), incluyendo el fortalecimiento de capacidades, desarrollo de habilidades y movilización comunitaria de trabajadoras sexuales (Jana et al. 1998; Campbell et al. 2001; Evans 1999; Busza and Schunter 2001); un mayor involucramiento de personas claves, como dueños y administradores de establecimientos de sexo (Visrutaratna et al. 
1995; Sakondhavat et al. 1997; Morisky et al. 1998; Morisky et al. 2002); y actividades educativas con clientes masculinos (Leonard et al. 2000).

\section{Programa Tailandés 100 Por Ciento Condón}

Estos esfuerzos ganaron atención considerable como resultado del programa tailandés 100 por ciento Condón patrocinado por el gobierno, el cual requirió el uso de condones en todos los actos sexuales realizados en burdeles (Rojanapithayakorn and Hanenberg 1996). El programa incluyó además acceso gratuito a condones en establecimientos de sexo, chequeo mensual de ITS en trabajadoras sexuales y una campaña nacional en los medios de comunicación dirigida a clientes masculinos de trabajadoras sexuales. Los dueños de establecimientos de sexo que no cumplieron con la política fueron sujetos a sanciones como multas y clausura.

Datos evaluativos del gobierno tailandés demuestran aumentos significativos en el uso de condones reportado en trabajadoras sexuales y sus clientes y reducciones significativas en las tasas de ITS en hombres que visitaron establecimientos de sexo durante el período 1989 a 1993 (Hanenberg et al. 1994). Investigaciones posteriores han confirmado un descenso en la incidencia de VIH en reclutas militares tailandeses quienes reportaron haber visitado establecimientos de sexo y quienes recibieron actividades educativas específicas (Mason et al. 1995; Nelson et al. 1996; Celentano et al. 1998; Nelson et al. 2002).

Los discursos actuales en la literatura científica han planteado una preocupación sobre el nivel de participación comunitaria en el Programa Tailandés 100 por ciento Condón además de su adaptabilidad a otros contextos culturales (Kilmarx et al. 1998; Evans 1999; Parker et al. 2000). Aunque varios programas y países han tratado de adoptar una estrategia regulativa parecida al programa Tailandés para prevenir el VIH/ITS en trabajadoras sexuales y sus parejas (UNAIDS 2002) esfuerzos para aumentar la participación comunitaria en el desarrollo de programa e implementación han sido más limitados.

\section{Adaptando el Programa Tailandés a la RD}

Por muchos años, dos ONGs dominicanas, Centro de Orientación e Investigación Integral (COIN) y Centro de Promoción y Solidaridad Humana (CEPROSH), han implementado estrategias de educación entre pares, distribución de condones, mejoramiento de manejo de ITS y movilización comunitaria y empoderamiento para prevenir el VIH/ITS en trabajadoras sexuales y sus clientes. Utilizando estas estrategias, el uso consistente de condones con clientes nuevos aumentó significativamente en áreas de intervención en ambas ciudades durante la última década.

Sin embargo, las tasas del uso consistente de condón con parejas fijas que pagan y que no pagan se han mantenido relativamente estable y significativamente más bajas que con clientes nuevos

(Rosario et al. 1994; CESDEM 1996; CESDEM 1999). Investigación cualitativa realizada en Santo Domingo revela que muchas trabajadoras sexuales reportaron que sus parejas fijas que pagan y que no pagan las ponen en mayor riesgo porque son menos propensas a usar condones con parejas de 


\section{Hgrizons}

confianza (Kerrigan et al. 2001). Este hallazgo sugiere que trabajadoras sexuales pueden necesitar más apoyo y motivación para negociar el uso de condones en estas relaciones de lo que proveyeron las estrategias de intervención anteriores.

A mediado de los 1990s, COIN y CEPROSH empezaron a explorar la adaptación del programa tailandés al contexto dominicano, incluyendo un mayor énfasis en la movilización comunitaria. Investigación etnográfica formativa realizada en Santo Domingo con trabajadoras sexuales y sus parejas, dueños y administradores de negocios de sexo y oficiales gubernamentales documentó suficiente apoyo para políticas gubernamentales y sistemas de apoyo para promover y monitorear el uso de condones en establecimientos de sexo (Kerrigan et al. 2001). Este estudio formativo también reveló las maneras en que las trabajadoras sexuales, dueños, administradores y otros empleados de los establecimientos ayudan y apoyan a las trabajadoras sexuales en las negociaciones de condones con clientes y que varios establecimientos ya habían desarrollados sus propias políticas institucionales de uso de condones (Kerrigan et al. 2001). Estos hallazgos confirmaron la necesidad de ir más allá que el modelo original tailandés de políticas y actividades patrocinadas por el gobierno para incluir más estrategias participativas y comunitarias para crear un ambiente que fomente el uso de condones en establecimientos de sexo.

Después, en colaboración con el gobierno dominicano, la Universidad de Johns Hopkins y el Programa Horizons, las dos ONGs desarrollaron y probaron dos modelos para promover el uso 100 por ciento de condones en establecimientos de sexo: (1) un modelo de solidaridad basado en la comunidad y (2) un modelo de solidaridad basado en la comunidad más política gubernamental y regulación. La investigación resultante examinó el impacto de estos dos modelos en el riesgo relacionado al VIH en trabajadoras sexuales. 


\section{Métodos}

\section{Contexto del estudio}

El sexo comercial o el intercambio de sexo por dinero no son ilegales en la República Dominicana, siempre y cuando la trabajadora sexual no sea menor de 18 años de edad. Dado que no existe una ley que prohíba explícitamente el sexo comercial en la República Dominicana, ni las trabajadoras sexuales ni sus clientes pueden ser procesados por la policía u oficiales legales. Sin embargo, la discriminación y acoso de las trabajadoras sexuales, especialmente las de la calle, ha sido documentado en el pasado.

Se encuentran trabajadoras sexuales y sus clientes en todo el país, particularmente en áreas urbanas como Santo Domingo y áreas turísticas como Puerto Plata. Se estima que el 80 por ciento de ellas trabajan en establecimientos de sexo, incluyendo casas de cita, bares, discos y cabarets (Pareja y Rosario 1992), mientras que las otras están ubicadas en la calle, parques u otras áreas.

En general, existen dos tipos de establecimiento de sexo en la República Dominicana: directos e indirectos. Establecimientos de sexo directos son más viejos y más tradicionales, por ejemplo los burdeles y las casas de cita. Normalmente los hombres van a un burdel explícitamente para buscar una mujer con quien tener relaciones sexuales. Siempre hay camas en el lugar, aunque el sexo también puede ocurrir afuera del establecimiento en un motel. En los establecimientos directos, casi siempre la única fuente de ingreso para la mujer es lo que gana por tener sexo con clientes. Actualmente, los burdeles componen una pequeña proporción de la industria de sexo en establecimientos en la República Dominicana. Por ejemplo, en Santo Domingo solo un 10 por ciento de todos los establecimiento de sexo son burdeles (COIN 1998). Los burdeles normalmente son más caros y exclusivos que los otros tipos de establecimiento de sexo y son visitados por hombres de un nivel socioeconómico alto.

Los establecimientos indirectos son los más comunes en el país; en Santo Domingo un 90 por ciento de todos los establecimientos de sexo son indirectos (COIN 1998). Lo que distingue los establecimientos indirectos de los directos es que contratan a las mujeres para proveer un servicio aparte del sexo, como camarera o bailarina (Kerrigan et al. 2001). Ejemplos de establecimientos indirectos incluyen bares, discos y clubes de baile desnudo. Muchas veces los establecimientos indirectos no tienen camas y las mujeres raramente viven en el lugar del negocio. El sexo con los clientes normalmente ocurre en lugares como moteles cercanos al establecimiento.

En el caso tanto de los establecimientos directos como los indirectos, los clientes pagan una tarifa fija, o salida, al dueño del negocio para tener relaciones sexuales con una mujer que trabaja en el mismo. La mujer negocia su propio precio con el cliente y se queda con este dinero. Otros establecimientos más informales también existen, donde no se paga salida a la casa pero las mujeres salen con hombres para tener relaciones sexuales. Sin embargo, el enfoque del actual estudio de intervención fue en los establecimientos donde se paga salida a la casa. 


\section{Hgrizons}

\section{Diseño del Estudio}

Para evaluar el impacto de los dos modelos de promoción de 100 por ciento uso de condones implementados durante un año en 34 establecimientos de sexo en Santo Domingo y Puerto Plata se utilizó un diseño pre/post prueba. Se complementó el diseño pre/post prueba con datos transversales sobre el nivel de cumplimiento con actividades de intervención recolectados mensualmente en cada establecimiento participando en el estudio. Las dos ciudades escogidas para participar en el estudio fueron seleccionadas sobre la base de varios criterios:

- El compromiso del nivel político para apoyar el estudio de intervención

- La capacidad técnica de las ONGs para llevar a cabo las actividades de intervención

- La existencia de un gran número de establecimientos de sexo

- La disponibilidad de un sistema público, viable de monitoreo y manejo de ITS

Dentro de cada ciudad, los investigadores seleccionaron áreas geográficas a propósito con la más alta prevalencia de sexo comercial determinadas por un censo realizado por las ONGs para implementar las actividades de intervención. Los criterios de selección para los establecimientos de sexo incluyeron que había más que 5 mujeres trabajando en el lugar y que el cliente pagaba salida al establecimiento. Estos criterios permitieron la inclusión de tanto establecimientos directos como indirectos. Todos los establecimientos de sexo que llenaron los criterios de selección dentro de las áreas geográficas establecidas fueron invitados a participar, y todos aceptaron.

\section{La I ntervención}

Los dos modelos de promoción de 100 por ciento condones fueron desarrollados después de una extensa investigación cualitativa y cuantitativa, y en consulta con trabajadoras sexuales líderes de pares y miembros de la organización local de trabajadoras sexuales, MODEMU. En Santo Domingo se implementó un modelo basado en la comunidad, enfocado en construir solidaridad entre trabajadoras sexuales, dueños de establecimientos de sexo y otras personas involucradas en el trabajo sexual, mientras que el modelo en Puerto Plata combinó esta estrategia comunitaria con una política gubernamental regional que requirió el uso de condones en todos establecimientos de sexo. Ambos modelos fueron estandarizados en las dos ciudades para el estudio de intervención.

Ambos modelos toman en cuenta la teoría ambiental-estructural, la cual propone que el cambio de comportamiento en individuos es facilitado o limitado por en ambiente físico, social y político donde se define las posibles opciones y donde se toma las decisiones (Sweat and Denison 1995). En el caso de la prevención de VIH, las trabajadoras sexuales frecuentemente son las únicas actrices responsables por la reducción de riesgo sin tomar en cuenta el desequilibrio de poder por género y nivel socioeconómico entre ellas, sus clientes y los dueños y administradores de establecimientos. Intervenciones basadas en la teoría ambiental-estructural tratan de crear un ambiente donde las trabajadoras sexuales no sean las únicas responsables sino donde todos los miembros de la industria del sexo sean mutuamente responsables para prevenir el VIH/ITS. 
Las ONGs y agencias gubernamentales implementaron las actividades de intervención. COIN en Santo Domingo y CEPROSH en Puerto Plata organizaron la investigación y los esfuerzos educativos y de construcción de solidaridad en cada ciudad, en colaboración con MODEMU. La Dirección General para el Control de las Infecciones de Transmisión Sexual y SIDA (DIGECITSS) y la Dirección Provincial de Salud en Puerto Plata tuvieron papeles claves en el desarrollo e implementación de la política de 100 por ciento condón implementado en Puerto Plata. El Instituto Dermatológico y Cirugía de la Piel y varios otros clínicos públicos de ITS realizaron chequeos periódicos de ITS y análisis biomédico como parte de las actividades de evaluación. El Departamento de Salud Ambiental, tanto en el nivel local como en el nivel nacional, ayudó en la evaluación del proyecto. Donantes internacionales, universidades y agencias, como el Programa Horizons, la Escuela de Salud Publica de Johns Hopkins, el proyecto Accion-SIDA del Academy for Educational Development, USAID/Washington, y USAID/República Dominicana, suministraron apoyo técnico y financiero para la intervención y la investigación.

Las siguientes secciones describen las actividades de intervención claves que fueron implementadas en ambas ciudades.

\section{Construyendo solidaridad y un compromiso colectivo}

El equipo del proyecto realizó varias actividades para construir solidaridad entre trabajadoras sexuales, dueños/administradores y otros empleados de los establecimientos de sexo para desarrollar un compromiso colectivo al uso 100 por ciento de condones entre trabajadoras sexuales y sus clientes. Estos esfuerzos incluyeron talleres participativos que dieron luz al papel de cada individuo en ayudar y apoyar a las trabajadoras sexuales en la negociación de condones con sus parejas sexuales.

Estos talleres fueron facilitados con cada uno de los tres grupos claves e incluyeron participantes de diferentes negocios. En total, se realizaron talleres cada tres meses con cada grupo durante el año de la intervención.

Se les pidió a todos los participantes en los talleres que firmaran un contrato social de su compromiso a promover la salud y bienestar del establecimiento. Materiales educativos fueron diseñados específicamente para cada grupo para reforzar la idea del compromiso colectivo y resaltar los derechos y deberes de cada grupo para proteger la salud y el bienestar de todos.

Otro tema en los talleres fue el papel de la confianza e intimidad entre las trabajadoras sexuales y sus parejas fijas que pagan y que no pagan y su efecto en el uso de condones y riesgo para el VIH. Investigación formativa

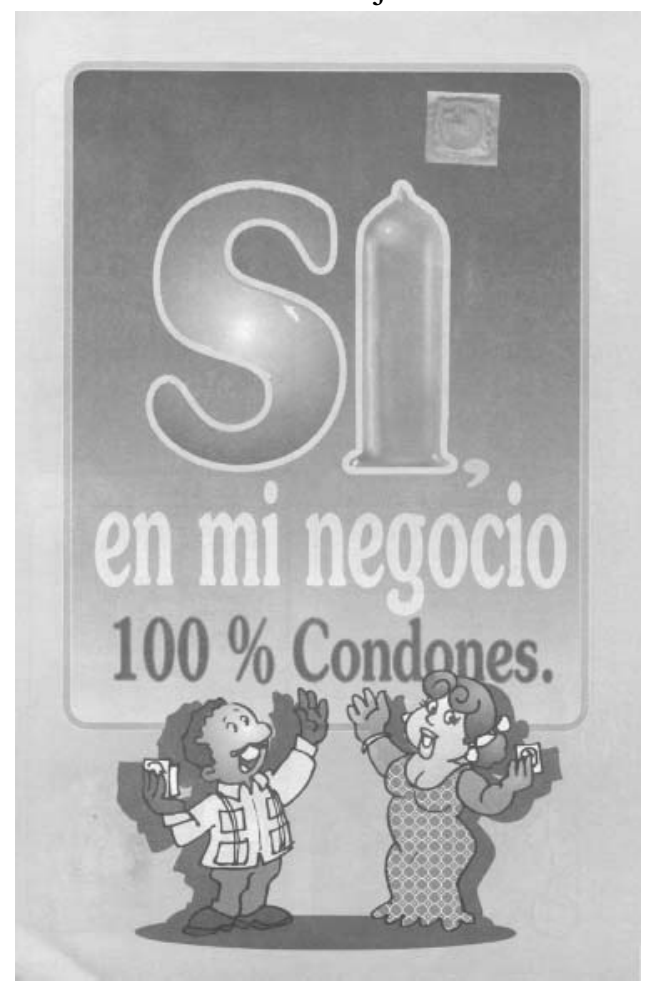




\section{Hgrizons}

reveló que existe un espectro de tipos de parejas sexuales para muchas trabajadoras sexuales, y no una dicotomía estricta entre cliente y novio o esposo que no paga (Kerrigan et al. 2001). Durante los talleres, dinámicas interactivas ayudaron a las mujeres a describir y definir los diferentes tipos de relaciones que tienen con sus parejas sexuales e identificar en conjunto los riesgos para el VIH/ITS asociados con cada tipo de relación. Como grupo, las mujeres hicieron lluvia de ideas sobre como podrían ser más críticas y objetivas en el establecimiento de relaciones de confianza y participaron en juegos de roles para desarrollar técnicas de comunicación y de negociación para procurar un sexo más seguro con los diferentes tipos de pareja. Mensajes y materiales educativos fueron diseñados específicamente para estos propósitos.

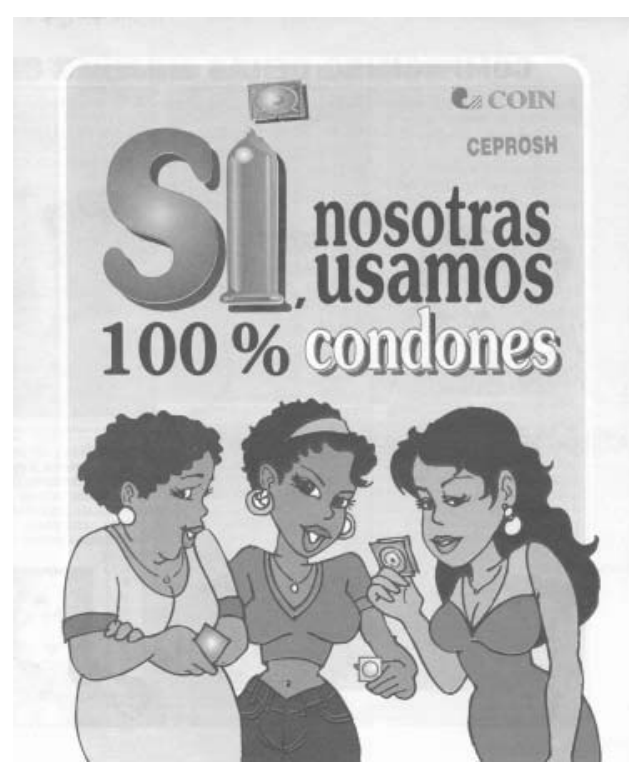

En el último taller, se animó a los participantes a compartir sus experiencias en las actividades de la intervención y proveer su retro-alimentación. También reuniones mensuales fueron realizadas en cada establecimiento para evaluar el progreso y discutir como superar las barreras al logro de los principios del compromiso colectivo con el uso de condones. Tantos los talleres cuatrimestrales como las reuniones mensuales fueron facilitados por personal de las ONGs y trabajadores sexuales educadoras de pares.

Cuando nuevas trabajadoras sexuales llegaron a los establecimientos durante el periodo de intervención, fueron invitadas al próximo taller, orientadas sobres los conceptos claves de las sesiones anteriores, solicitadas a firmar el contrato del compromiso colectivo y también recibieron materiales educativos y apoyo adicional, tanto como fuese necesario.

\section{Facilitando recordatorios ambientales a la acción}

En ambas ciudades, recordatorios ambientales promovieron el uso consistente de condones entre trabajadoras sexuales y sus clientes. Cada dueño de establecimiento fue animado a poner afiches y calcomanías promoviendo el uso 100 por ciento de condones y recipientes transparentes de vidrio llenos de condones en lugares visibles por todo el establecimiento; estos materiales fueron proveídos por el proyecto sin gasto al dueño. Todos los establecimientos tuvieron la responsabilidad de asegurar acceso a condones de alta calidad y de siempre mantener una reserva de por lo menos 100 condones en el establecimiento. Para apoyar este proceso, el proyecto vendió los condones a los dueños a bajo precio. En cambio, los dueños de negocios fueron animados a regalar por lo menos dos condones a las trabajadoras sexuales cuando hicieron una salida del negocio. Muchos establecimientos buscaron recuperar el costo de proveer los condones gratis a las trabajadoras sexuales con un leve aumento en el precio de la salida pagado por el cliente al establecimiento para salir con una trabajadora sexual. 
Otros recordatorios ambientales incluyeron que en algunas noches los disc jockeys dijeron mensajes sobre la importancia de los condones y el sexo más seguro. Al mismo tiempo, se reforzaron los mensajes de los disc jockeys con mesas de información en la entrada de los establecimientos, además de teatro participativo y materiales educativos para los clientes masculinos de las trabajadoras sexuales. El equipo de las ONGs y las trabajadoras sexuales educadoras de pares trabajaron con los disc jockey para desarrollar los mensajes, manejaron las mesas de información, participaron en las presentaciones de teatro y distribuyeron los materiales educativos.

\section{Promoviendo servicios clínicos respetuosos para las trabajadoras sexuales}

Antes de comenzar la intervención, el gobierno Dominicano ya tuvo el requisito de que todas las trabajadoras sexuales asistieran a un chequeo mensual de ITS en clínicas públicas del gobierno. Inspectores de salud del gobierno tuvieron la responsabilidad de asegurar que todas las trabajadoras sexuales asistieran a estos chequeos y revisar que todas las mujeres trabajando en establecimientos de sexo tuviesen una tarjeta de chequeo de ITS actualizada. Generalmente los chequeos mensuales fueron gratis, aunque se cobraba un poco para una tarjeta perdida (US\$2.50). Dado a los pocos recursos, organización y supervisión, el sistema de los chequeos mensuales no fue implementado de una manera estandarizada.

La intervención buscó mejorar el sistema primero con una evaluación de las necesidades de las clínicas públicas que dan servicios a trabajadoras sexuales y con entrevistas con los inspectores de salud del gobierno. Después, el personal de las ONGs facilitó capacitaciones para el personal clínico de las clínicas públicas y los inspectores de salud ambiental sobre el VIH/SIDA, la recolección de datos y monitoreo, los derechos humanos de las trabajadoras sexuales y los estándares éticos y procedimientos relacionados con la confidencialidad. Este proyecto también ayudó a los trabajadores de salud gubernamentales en abogar con éxito al gobierno nacional para adquirir equipamiento, personal y medicinas para el manejo de ITS. Adicionalmente, varias trabajadoras sexuales educadoras de pares del proyecto recibieron por primera vez oficinas privadas en las clínicas públicas para proveer conserjería como parte del manejo de ITS y pruebas voluntarias del VIH. Ellas también ayudaron a los inspectores de salud para recordar a los dueños y administradores de los establecimientos del día y la hora del próximo chequeo de ITS para las trabajadoras sexuales.

\section{Monitoreando y animando el cumplimiento}

En el comienzo del proyecto, la dirección provincial de salud de Puerto Plata y la ONG CEPROSH anunciaron la política del uso 100 por ciento de condones en un evento atendido por los dueños de los 34 establecimientos de sexo participantes en el evento. Se informó a los dueños que ellos, y no las trabajadoras sexuales, eran los responsables para asegurar que el establecimiento cumpliese con la política del uso 100 por ciento de condones y las actividades del programa. El personal de la ONG y las autoridades gubernamentales en Puerto Plata se reunieron con los dueños de estos 


\section{Hgrizons}

establecimientos cada tres meses para motivar su colaboración continua y para discutir las barreras al cumplimiento.

Tanto en Puerto Plata como en Santo Domingo, los inspectores de salud gubernamentales acompañados por personal de las ONGs visitaron los establecimientos de sexo de manera mensual para documentar y notificar a los dueños sobre su nivel de cumplimiento con los cinco elementos claves:

- La presencia de afiches promoviendo el uso 100 pro ciento de condones.

- Disponibilidad de por lo menos 100 condones.

- Condones visible y accesible en el negocio.

- Asistencia de todas las trabajadoras sexuales al chequeo mensual de ITS.

- Ningún diagnóstico positivo de ITS en trabajadoras sexuales trabajando en el negocio. No se compartieron los resultados de las pruebas de ITS de trabajadoras sexuales con los dueños de los establecimientos. Ellos simplemente fueron informados si su establecimiento estuvo cumpliendo, lo cual significaba que no tenían un diagnóstico positivo para ITS en las mujeres trabajando en el negocio durante el mes.

El propósito de estas visitas mensuales de monitoreo fue garantizar una retroalimentación continua y apoyo a todos los establecimientos participantes en la intervención. Al final de cada mes, los establecimientos en ambas ciudades que no cumplieron con los cinco elementos recibieron una educación más intensa para mejorar el cumplimiento. Además de estos esfuerzos educativos intensificados, los establecimientos no cumplidores con los cinco elementos en Puerto Plata fueron sujetos a un sistema de sanciones graduadas orientado hacia el dueño, incluyendo notificaciones, multas y clausuras. En ambas ciudades, cada tres meses se otorgó certificados a los establecimientos que cumplieron con los cinco elementos.

\section{Recolección de Datos y Procedimientos Éticos}

Dos comités de ética independientes en los Estados Unidos revisaron y aprobaron el protocolo de la investigación: Population Council en Nueva York y la Universidad de Johns Hopkins en Baltimore, Maryland. Adicionalmente, DIGECITSS revisó y aprobó el estudio con relación a su mérito técnico y ético.

El equipo de la investigación recolectó una línea de base de datos evaluativos (septiembre a diciembre 1999) y otra vez al final del periodo de intervención de 12 meses (noviembre a diciembre 2000). Las fuentes de los datos fueron auto-reporte, observación y pruebas clínicas. Los datos fueron triangulados como parte del análisis para responder a las preguntas de investigación. Una muestra transversal, al azar de aproximadamente 200 trabajadoras sexuales de establecimientos de sexo participando en la intervención completó encuestas sociales y de comportamiento administrado por entrevistadores capacitados de ONGs de salud locales. Esta muestra de trabajadoras sexuales también recibió pruebas de ITS no-rutinarias antes y después de la intervención, específicamente, muestras de orina para gonorrea y Clamidia y muestras vaginales para Tricomoniasis. 
Las participantes fueron reclutadas en las clínicas públicas donde las trabajadoras sexuales asisten a sus chequeos mensuales de ITS. Cada tercera trabajadora sexual examinada por los médicos de la clínica como parte de su atención rutinaria fue invitada a participar en una entrevista preliminar en un lugar privado. Se realizó un proceso de consentimiento informado con todas las mujeres que mostraron interés inicial y llenaron los criterios de selección del estudio: 18 años de edad o más y que actualmente trabaja en uno de los 34 establecimientos de sexo participantes en la intervención. Todas las potenciales participantes recibieron información detallada sobre los objetivos, procedimientos y riesgos y beneficios del estudio. Si aceptaron participar en el estudio, dieron su consentimiento por escrito para ser entrevistada y para participar en un chequeo de ITS norutinaria. Todas las participantes recibieron aproximadamente US $\$ 3$ por su tiempo. La tasa de participación en el estudio fue por encima del 95 por ciento.

Todas las encuestas fueron anónimas y la prueba de ITS fue confidencial. Los nombres de las personas fueron anotadas con los números de record de las participantes en un lugar aparte de las encuestas. Se explicó a las participantes que deberían volver dentro de una semana para recibir sus resultados de ITS y tratamiento gratis en caso de que tuviesen una prueba positiva. Educadoras de pares de las ONGs contactaron de manera confidencial a las participantes que tuvieron una prueba positiva pero que no volvieron después de una semana y les dieron tratamiento para su ITS.

También fueron realizadas observaciones participativas pre- y post-intervención en una muestra al azar de 64 trabajadoras sexuales reclutadas de establecimientos de sexo participando en la intervención en ambas ciudades. El equipo del estudio seleccionó personal masculino de las ONGs para pasar como clientes masculinos y los capacitaron en la metodología de observación participativa y ética de investigación. Después de seleccionar las mujeres sobre la base de criterios establecidos, el personal de la ONGs siguió un protocolo de investigación estricto: hablaron con la mujer por aproximadamente 30 minutos y después preguntaron si ella estaría de acuerdo en tener relaciones sexuales sin usar un condón, utilizando hasta 4 razones por la cual no se debe usar un condón (ver abajo las cuatro razones especificas). Después que la trabajadora sexual daba su última respuesta, el pidió excusa, pagó la cuenta de su mesa y compensó a la trabajadora sexual con una propina de aproximadamente US $\$ 6$ por su tiempo. Todas las interacciones fueron anónimas y tuvieron lugar dentro de los establecimientos. Después de salir del establecimiento, el personal de las ONGs documentó la interacción en un lugar privado. Resultados no fueron compartidos con nadie fuera del equipo del estudio. La organización local de trabajadoras sexuales, MODEMU, fue consultada sobre las observaciones y aprobó la metodología de observación como parte de las actividades de evaluación.

Además de los datos al nivel individual que fueron recolectados pre- y post-intervención, también fueron recolectados datos de cada establecimiento de sexo mensualmente para documentar el cumplimiento con los cinco elementos claves de intervención, como fueron descritos arriba. 


\section{Hgrizons}

\section{Medidas}

El uso de condón fue evaluado en las encuestas por auto-reporte de las participantes del por ciento de sus actos sexuales en los cuales usaron condones en el último mes con nuevos clientes, parejas fijas que pagan y que no pagan y todas las parejas. Los clientes nuevos fueron definidos como personas con las que tuvieron relaciones sexuales por dinero solo una o dos veces. Las parejas fijas fueron definidas como personas con las que tuvieron relaciones por lo menos tres veces o con quien tuvieron una relación de confianza, si pagaran por el sexo o no. Entonces, la categoría de pareja fija incluyó tantos parejas que pagan como las parejas que no pagan. Se utilizó una escala Likert de 5 puntos para medir la frecuencia del uso de condón con los clientes nuevos y las parejas fijas: siempre, casi siempre, a veces, casi nunca y nunca. Con relación a todas las parejas, se les preguntó a las participantes con cuantas parejas habían tenido relaciones en el último mes y con cuantas de estas siempre habían usado condones.

El rechazo al sexo inseguro fue medido por observaciones participativas de las trabajadoras sexuales por personal de las ONG pasando como clientes masculinos. Una investigación formativa había revelado las excusas comunes dada por los hombres para no querer usar condones con trabajadoras sexuales. El equipo de investigación seleccionó cuatro razones para ser usadas por los clientes secretos: no le gustan los condones, es un "tipo serio que está casado y tiene hijos", dará 50 por ciento más dinero de lo que pide ella y dará 100 por ciento más dinero de lo que pide ella. El personal de las ONGs pasando como un cliente masculino anotó si la trabajadora sexual aceptó alguna de estas razones o si verbalmente rechazó el sexo inseguro durante el encuentro.

Las infecciones de transmisión sexua (ITS)l fueron documentadas calculando la prevalencia de cada una de las tres ITS curables evaluadas por el estudio en trabajadoras sexuales: gonorrea, Clamidia y Tricomoniasis. Después se creó una medida dicotomizada de que si la participante tenía alguna de las tres ITS. Clamidia y gonorrea fueron detectadas usando Ligase chain reaction (LCR) pruebas de ADN, mientras la presencia de Tricomoniasis fue establecida utilizando pruebas de cultivo. El tratamiento fue proveído a todas las mujeres que tenían pruebas positivas por cualquier de las ITS en el pre- y post-test.

Exposición a la intervención: Dentro de la encuesta pre-post realizada con trabajadoras sexuales en cada ciudad, se midió la exposición reportada a la intervención utilizando una escala de 13 puntos (Cronbach's alpha $=.80)$, incluyendo la participación, exposición y/o percepciones de la trabajadora sexual sobre los componentes claves de la intervención en el último mes, incluyendo los siguientes elementos:

- Compromiso colectivo: participó en talleres de construcción de solidaridad y recibió materiales de educación, firmó un acuerdo del compromiso colectivo, sintió apoyo o ánimo de parte del dueño/administrador, empleados u otras trabajadoras sexuales sobre el uso de condones.

- Recordatorios ambientales: vio afiches o condones en el establecimiento y tuvo acceso siempre a condones en el establecimiento.

- Política: escuchó al dueño del establecimiento comunicar una política clara sobre el uso 100 por ciento de condones y/o supo de una reunión facilitada por el dueño para reforzar esta política o norma. 
- Monitoreo: asistió al chequeo de ITS mensual y/o vio a un inspector de salud gubernamental visitar el establecimiento para confirmar que los chequeos estuvieran al día.

La escala de exposición reportada después fue dicotomizada en el punto mediano de su distribución en alta exposición (repuesta positiva a 11 elementos o más) versus baja exposición (respuesta positiva a 10 elementos o menos).

Cumplimiento con la intervención: Una medida del nivel de cumplimiento observado con la intervención fue calculada usando datos de los chequeos ambientales mensuales realizados en cada establecimiento durante los 12 meses de la intervención. Cada establecimiento recibió una puntuación de 0 a 5 por cada mes dependiendo de su cumplimiento con los 5 elementos claves de la intervención en el último mes, incluyendo: (1) la presencia de afiches de 100 por ciento uso de condones en el establecimiento, (2) condones visibles en recipientes transparentes de vidrio u otros recipientes dentro del establecimiento, (3) una reserva de por lo menos 100 condones en el establecimiento, (4) asistencia de todas la trabajadoras sexuales a los chequeos mensuales de ITS, y (5) ausencia de un diagnóstico positivo de una ITS en las trabajadoras sexuales trabajando en el negocio. Después, se calculó el nivel promedio de cumplimiento con estos 5 elementos durante el periodo de un año de intervención por establecimiento el cual fue aplicado a cada participante de cada establecimiento para fines de los análisis de regresión.

Información sociodemográfica, como edad en años, número de años cumplidos en la escuela, estado civil (casada o en unión versus soltera o divorciada), número de salidas con clientes en la semana pasada, número total de parejas sexuales en el último mes y la tarifa promedio cobrada a cada cliente por salida, también fue recolectada dentro del contexto de las encuestas pre-y post-test con las trabajadoras sexuales.

\section{Manejo de Datos y Análisis}

El análisis de los datos fue realizado utilizando dos paquetes: SPSS 11.0 (C) para Windows (univariable y bivariable) y STATA Intercooled Version 7.0 () (multivariable). Los análisis univariables incluyeron frecuencias y distribuciones de las variables del estudio realizadas con los datos tanto de la encuesta pre-intervención como de la post-intervención. Las variables continuas fueron examinadas por la normalidad de su distribución y fueron clasificadas en categorías sobre la base de sus distribuciones en el punto mediano. Todas las variables relacionadas al uso de condones fueron entonces dicotomizadas entre el uso consistente de condones (siempre o con todas las parejas) versus el uso inconsistente (menos que siempre o con menos que todas las parejas). Análisis bi-variables incluyeron pruebas de asociación de Chi y pruebas $t$ de muestras independientes. Estas pruebas fueron realizadas para evaluar si existían diferencias estadísticamente significativas entre las muestras pre- y post-intervención con relación a los resultados relacionados con el riesgo del $\mathrm{VIH}$, exposición a la intervención, cumplimiento con la intervención y características sociodemográficas.

Los análisis de regresión bi-variables y multi-variables fueron realizados con los datos de trabajadoras sexuales que reportaron haber tenido una pareja o más en el último mes, utilizando 


\section{Hgrizons}

datos de post-intervención para determinar la probabilidad no ajustada y ajustada del uso consistente de condones con todas las parejas en el último mes por el nivel de exposición a la intervención y el nivel de cumplimiento con la intervención (controlado por otros factores sociodemográficos). Los análisis de regresión bi-variables y multi-variables fueron realizados tanto incluyendo como excluyendo casos con información faltante. No se detectó diferencias estadísticamente significativas en los resultados de estos análisis; entonces, los 42 casos postintervención con información faltante fueron excluidos del último análisis de regresión.

Se ajustaron los errores estándares de los análisis de regresión para el posible "efecto de conglomerado" en los datos reportados de uso de condones entre mujeres del mismo establecimiento de sexo. Estos ajustes fueron hechos utilizando el coeficiente de correlación robusto de Huber-White. Todas las variables incluidas en el último modelo de regresión multivariable fueron probadas por co-linealidad antes de su inclusión en el modelo. 


\section{Resultados}

\section{Características de la Muestra}

La edad mediana de las trabajadoras sexuales participando en la encuesta pre-intervención fue de aproximadamente 25 años (Tabla 1). El porcentaje de mujeres por encima de los 25 años no varió significativamente entre ciudades ( $\mathrm{p}<0.167)$. Entre mujeres en ambas ciudades, el nivel mediano de escolaridad cumplido fue de 7.0 años. El porcentaje de mujeres que cumplieron más allá que la escuela primaria (> 8 años) no varió significativamente entres las ciudades ( $\mathrm{p}<0.378$ ). Mientras que más que el 70 por ciento de mujeres en ambas ciudades reportaron su estado civil oficial como soltera, más del 65 por ciento de las mujeres de cada ciudad reportaron tener una pareja fija actualmente. Ni el estado civil ( $p<0.901)$, ni el tener una pareja fija $(\mathrm{p}<0.328)$ variaron significativamente entre las dos ciudades.

El número mediano de citas con clientes en la última semana en las trabajadoras sexuales de Santo Domingo fue 1.0 (0-30), comparado con 2.0 (0-32) en las trabajadoras sexuales de Puerto Plata. El número total mediano de parejas sexuales en el último mes fue 2.0 (0-40) en mujeres de Santo Domingo y $3.0(0-50)$ en mujeres de Puerto Plata. El porcentaje de mujeres reportando tres parejas sexuales o más en el último mes varió significativamente entre las dos ciudades $(\mathrm{p}<0.000)$, mientras que el 24.9 por ciento de mujeres en Santo Domingo reportaron haber tenido tres parejas sexuales o más en el último mes, fue del 49.7 por ciento en Puerto Plata. La cantidad promedio mediana de dinero cobrado por cita con un cliente fue US\$18 en participantes de las dos ciudades. Sin embargo, el porcentaje de mujeres cobrando más que US\$18 por cita varió significativamente por ciudad ( $\mathrm{p}<0.002$ ), con un porcentaje más alto de trabajadoras sexuales en Santo Domingo, 42.9 por ciento, cobrando US $\$ 19$ o más por cita que en Puerto Plata, donde fue el 28.5 por ciento.

Algunas características sociodemográficas variaron significativamente entre la encuestas pre- y post-intervención ( $\mathrm{p}$ < 0.05). En Santo Domingo, había más mujeres casadas en la muestra de la encuesta post-intervención que en la encuesta pre-intervención. En Puerto Plata, más mujeres tenían niveles más bajos de educación, más de dos citas en la última semana y actualmente tenían una pareja fija en la encuesta post-intervención comparada con la encuesta pre-intervención. En ambas ciudades, había más participantes con más que tres parejas sexuales en el último mes en el post-test comparado con el pre-test. 
Tabla 1 Características sociodemográficas y de comportamiento en encuesta pre-test

\begin{tabular}{|c|c|c|c|}
\hline \multirow[t]{2}{*}{ Variables } & \multicolumn{2}{|c|}{ Mediano/rango y frecuencias } & \multirow[t]{2}{*}{ Valor $p$} \\
\hline & $\begin{array}{l}\text { Santo Domingo } \\
(n=210)\end{array}$ & $\begin{array}{l}\text { Puerto Plata } \\
(\mathrm{n}=200)\end{array}$ & \\
\hline Edad en años & $24.0(18-40)$ & $25.0(18-51)$ & 0.167 \\
\hline $18-25$ & 60.8 & 54.0 & \\
\hline $26+$ & 39.2 & 46.0 & \\
\hline Educación en años & $7.0(0-13)$ & $7.0(0-12)$ & 0.378 \\
\hline $0-8$ & 69.0 & 73.0 & \\
\hline $9+$ & 31.0 & 27.0 & \\
\hline Estado civil & & & 0.901 \\
\hline Soltera & 72.1 & 71.5 & \\
\hline Casada & 27.9 & 28.5 & \\
\hline Actualmente tiene una pareja fija & & & 0.328 \\
\hline Sí & 68.6 & 64.0 & \\
\hline No & 31.4 & 36.0 & \\
\hline $\begin{array}{l}\text { Número de citas con clientes en la } \\
\text { última semana }\end{array}$ & $1(0-30)$ & $2(0-32)$ & 0.020 \\
\hline$<2$ & 56.5 & 44.7 & \\
\hline $2+$ & 43.5 & 55.3 & \\
\hline $\begin{array}{l}\text { Número total de parejas sexuales en el } \\
\text { último mes }\end{array}$ & $1(0-40)$ & $2(0-50)$ & 0.000 \\
\hline$<3$ & 75.1 & 50.3 & \\
\hline $3+$ & 24.9 & 49.7 & \\
\hline $\begin{array}{l}\text { Tarifa promedia cobrada por cita con } \\
\text { cliente }\end{array}$ & $\$ 18(12-90)$ & $\$ 18(12-60)$ & 0.002 \\
\hline$\$ 0-18$ & 57.1 & 71.5 & \\
\hline$\$ 19+$ & 42.9 & 28.5 & \\
\hline
\end{tabular}

\section{Cambios Pre- a Post-intervención en Riesgo Relacionado al VI H}

\section{Uso consistente de condones}

El uso consistente de condones (UCC) reportado con clientes nuevos en el último mes en la encuesta pre-intervención fue 75.3 por ciento en Santo Domingo, comparado con 96.5 por ciento en Puerto Plata (Tabla 2). La tasa de UCC con clientes nuevos aumentó en las participantes en la encuesta post-intervención comparado con la pre-intervención en ambas ciudades, a 98.3 por ciento en Santo Domingo y a 98.6 por ciento en Puerto Plata. Sin embargo, dado que la tasa del uso de condones con clientes nuevos ya fue muy alta en la encuesta pre-intervención en Puerta Plata, este cambio solo fue estadísticamente significativo en Santo Domingo $(\mathrm{p}<0.000)$. 
En el caso de las parejas fijas que pagan y no pagan, las tasas de UCC en la encuesta preintervención fueron parecidas en las dos ciudades: 14.6 por ciento en Santo Domingo versus 13.0 por ciento en Puerto Plata. Sin embargo, la tasa de UCC con parejas fijas en el último mes en la encuesta post-test aumentó de manera significativa solamente en Puerto Plata, llegando a un 28.8 por ciento $(\mathrm{p}<0.001)$.

\begin{tabular}{|c|c|c|c|c|c|c|c|c|c|c|c|c|}
\hline \multirow{3}{*}{$\begin{array}{l}\text { Tipo de } \\
\text { pareja }\end{array}$} & \multicolumn{6}{|c|}{ Santo Domingo } & \multicolumn{6}{|c|}{ Puerto Plata } \\
\hline & \multicolumn{2}{|c|}{ Pre } & \multicolumn{2}{|c|}{ Post } & \multicolumn{2}{|c|}{ Valor $p$} & \multicolumn{2}{|c|}{ Pre } & \multicolumn{2}{|c|}{ Post } & \multicolumn{2}{|c|}{ Valor $\mathbf{p}$} \\
\hline & $\%$ & $\mathbf{n}$ & $\%$ & $\mathbf{n}$ & & $\mathbf{n}$ & $\%$ & $\mathbf{n}$ & $\%$ & $\mathbf{n}$ & & $\mathbf{n}$ \\
\hline $\begin{array}{l}\text { Clientes } \\
\text { nuevos }\end{array}$ & 75.3 & 93 & 93.8 & 113 & 0.000 & 206 & 96.5 & 115 & 98.6 & 141 & 0.279 & 256 \\
\hline $\begin{array}{l}\text { Parejas } \\
\text { fijas }\end{array}$ & 14.6 & 144 & 17.6 & 165 & 0.476 & 309 & 13.0 & 131 & 28.8 & 153 & 0.001 & 284 \\
\hline
\end{tabular}

Las "n" en la Tabla 2 representan el número de participantes que reportaron haber tenido estos tipos de pareja en el último mes, quienes después fueron preguntados y dieron respuesta de que si usaron condones de manera consistente con estas parejas.

\section{Rechazo verbal al sexo inseguro}

La habilidad de las trabajadoras sexual para rechazar el sexo comercial inseguro aumentó de pre- a post-intervención en ambas ciudades. Sin embargo, aumentos estadísticamente significativos solo fueron documentados en Puerto Plata (Tabla 3). Estos aumentos fueron encontrados en cada uno de los cuatro niveles de interacción social evaluada por el estudio. El porcentaje total de trabajadoras sexuales con la habilidad de rechazar verbalmente el sexo inseguro después de los cuatro escenarios que fueron presentados aumentó en casi un 30 por ciento, desde el 50.0 por ciento en la pre-intervención al 79.4 por ciento en la post-intervención en Puerto Plata ( $\mathrm{p}<0.000)$. En Santo Domingo, la habilidad de rechazar verbalmente el sexo inseguro aumentó desde el 64.2 por ciento al $72.3(\mathrm{p}<0.316)$. 
Tabla 3 Rechazo verbal del sexo inseguro, pre- a post-intervención

\begin{tabular}{lccc|ccc}
\hline $\begin{array}{l}\text { \% acumulativo } \\
\text { rechazando el sexo } \\
\text { inseguro por nivel }\end{array}$ & $\begin{array}{c}\text { Pre- } \\
\mathbf{n = 6 7}\end{array}$ & $\begin{array}{c}\text { Post- } \\
\mathbf{n = 6 5}\end{array}$ & $\begin{array}{c}\text { Valor } \mathbf{p} \\
\mathbf{n = 1 3 2}\end{array}$ & $\begin{array}{c}\text { Pre- } \\
\mathbf{n = 6 4}\end{array}$ & $\begin{array}{c}\text { Post- } \\
\mathbf{n = 6 8}\end{array}$ & $\begin{array}{c}\text { Valor } \mathbf{p} \\
\mathbf{n}=\mathbf{1 3 2}\end{array}$ \\
\hline $\begin{array}{l}\text { Dijo que no le gusta los } \\
\text { condones }\end{array}$ & 76.1 & 78.5 & 0.748 & 79.7 & 94.1 & 0.013 \\
Dijo que era un "tipo serio" & 71.6 & 76.9 & 0.488 & 64.1 & 91.2 & 0.000 \\
Ofreció 50\% más dinero & 67.2 & 73.8 & 0.400 & 54.7 & 83.8 & 0.000 \\
Ofreció 100\% más dinero & 64.2 & 72.3 & 0.316 & 50.0 & 79.4 & 0.000
\end{tabular}

\section{Infecciones de transmisión sexual}

Se detectaron reducciones significativas en la prevalencia de ITS (gonorrea, Tricomoniasis,y/o Clamidia) medida por el estudio en ambas ciudades, con una reducción levemente más fuerte en Puerto Plata (28.8 a 16.3 por ciento, p < 0.003), comparado con Santo Domingo (25.5 a 15.9 por ciento, $\mathrm{p}<0.014)$. Reducciones en las distintas ITS parecen estar empujando las reducciones generales en los niveles de prevalencia en ambas ciudades. En el caso de Santo Domingo, la prevalencia de Clamidia bajó significativamente desde el 16.4 por ciento al 9.3 por ciento ( $\mathrm{p}<0.029)$, mientras que en Puerto Plata la prevalencia de Tricomoniasis bajó significativamente de pre- a post-intervención, desde el 9.6 por ciento al 3.9 por ciento $(\mathrm{p}<0.024)$.

Tabla 4 Infecciones de transmisión sexual (ITS), pre- a post-intervención

\begin{tabular}{lccc|rrr}
\hline Tipo de ITS & \multicolumn{3}{c|}{ Santo Domingo } & \multicolumn{3}{c}{ Puerto Plata } \\
& $\begin{array}{l}\text { Pre- } \\
\mathbf{n = 2 2 0}\end{array}$ & $\begin{array}{c}\text { Post- } \\
\mathbf{n = 2 1 4}\end{array}$ & $\begin{array}{c}\text { Valor } \mathbf{~} \\
\mathbf{n = 4 3 4}\end{array}$ & $\begin{array}{c}\text { Pre- } \\
\mathbf{n = 1 9 8}\end{array}$ & $\begin{array}{c}\text { Post- } \\
\mathbf{n = 2 0 4}\end{array}$ & $\begin{array}{c}\text { Valor } \mathbf{p} \\
\mathbf{n}=\mathbf{4 0 2}\end{array}$ \\
\hline Gonorrea & 2.3 & 1.9 & 0.768 & 6.6 & 3.9 & 0.234 \\
Tricomoniasis & 9.1 & 6.1 & 0.236 & 9.6 & 3.9 & 0.024 \\
Clamidia & 16.4 & 9.3 & 0.029 & 14.6 & 9.8 & 0.138 \\
Una o más de las tres & 25.5 & 15.9 & 0.014 & 28.8 & 16.3 & 0.003 \\
ITS & & & & & &
\end{tabular}

\section{Exposición y cumplimiento con la intervención}

En ambas ciudades, la exposición reportada a la intervención en las fases de recolección de datos pre-intervención versus el post-intervención aumentó significativamente, con puntuaciones promedias en una escala de exposición de 13 puntos aumentando desde el 6.6 al 10.21 en Santo Domingo ( $\mathrm{p}<0.000)$ y desde el 9.5 al 11.72 en Puerto Plata ( $<$ 0.000). Sin embargo, el cumplimiento con la intervención se mostró de manera muy diferente en las dos ciudades. 
La Figura 1 demuestra el nivel promedio de cumplimiento con los cinco elementos ambientales claves observados de manera mensual durante el período de 12 meses de la intervención: (1) afiches visibles promoviendo 100 por ciento condones, (2) una recipiente transparente de vidrio llena de condones, (3) una reserva de al menos 100 condones en el establecimiento, (4) todas las mujeres asistiendo a los chequeos mensuales de ITS, y (5) ninguna mujer con una prueba positiva para una ITS. De acuerdo a estos datos, el nivel promedio de cumplimiento observado aumentó de manera más constante e intensa en Puerto Plata que en Santo Domingo. El nivel promedio de cumplimiento con estos cinco elementos de intervención en los establecimiento en Santo Domingo fue 3.3 en el mes 1, y terminó en casi el mismo nivel, 3.0 en el mes 12 de la intervención. En los establecimientos de Puerto Plata, el nivel promedio de cumplimiento con los cinco elementos claves de la intervención empezó en 2.6 en el mes 1 y aumentó significativamente a 4.7 en el mes 12 de la intervención $(\mathrm{p}<0.000)$.

Figura 1 Nivel promedio de cumplimiento con elementos ambientales de la intervención observado en los establecimientos en Santo Domingo (SD) y Puerto Plata (PP) (n = 68)

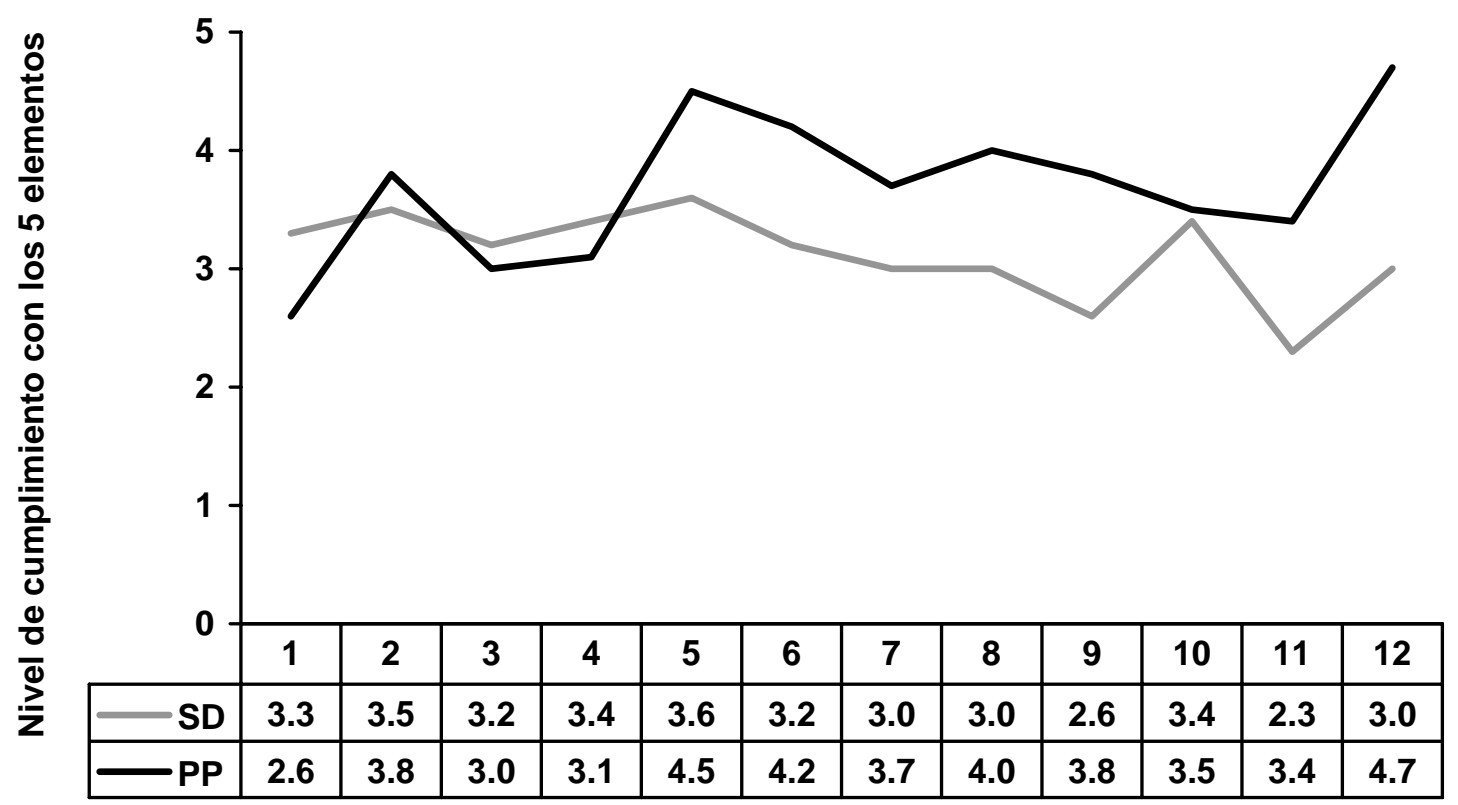

\section{Mes de la Intervencion}

Nota: Los cinco elementos incluyeron: (1) la presencia de afiches visibles promoviendo 100 por ciento condones en el establecimiento, (2) condones visibles en recipientes transparentes de vidrio dentro del establecimiento, (3) una reserva de al menos 100 condones en el establecimiento, (4) asistencia de todas las trabajadoras sexuales del establecimiento a los chequeos mensuales de ITS y (5) ningún diagnóstico positivo para una ITS en las trabajadoras sexuales trabajando en el establecimiento. 


\section{Hgrizons}

\section{Evaluando los efectos de exposición y cumplimiento con la intervención en el uso consistente de condones}

La Tabla 5 demuestra las probabilidades no-ajustadas y ajustadas del UCC con todas las parejas en el último mes dependiendo del nivel de exposición a la intervención, cumplimiento con la intervención, además de otros factores sociodemográficos que podrían estar asociados con el UCC en trabajadoras sexuales en ambas ciudades. Análisis bi-variable demuestra que tanto la exposición reportada a la intervención como el cumplimiento observado con la intervención están asociados significativamente con un aumento en el uso del condón. En trabajadoras sexuales con niveles más altos de exposición reportada a la intervención, la probabilidad del UCC fue 1.99 veces mayor que en trabajadoras sexuales con niveles más bajo de exposición. El UCC fue 3.76 veces mayor en trabajadoras de establecimientos con el más alto nivel de cumplimiento observado con los componentes claves de la intervención, comparado con las mujeres con niveles más bajos de cumplimiento observado.

En el análisis bi-variable, se encontró una asociación significativa también entre el haber reportado tener más que dos citas con clientes en la última semana y un aumento en el nivel de UCC. Las variables asociadas significativamente con el uso inconsistente de condones con todas las parejas en el último mes incluyeron estar casada o en unión y el tener una pareja fija actualmente. Adicionalmente, la ciudad donde trabajaban las trabajadoras sexuales también fue un determinante significativo del UCC. Mujeres de Puerto Plata, por ejemplo, fueron 2.29 veces más probable de usar condones de manera significativa con todas las parejas en el último mes que las mujeres de Santo Domingo, en los análisis bi-variables.

En el análisis multi-variable, la exposición a la intervención y el cumplimiento se mantienen asociados significativamente con el UCC. Las participantes en el estudio con altos niveles de exposición reportada a la intervención fueron 1.84 veces más probable de usar condones de manera consistente, comparada con mujeres con bajos niveles de exposición reportada. Participantes trabajando en establecimientos con los más altos niveles de cumplimiento observado con la intervención fueron 2.33 veces más probable a usar condones de manera consistente, comparada con mujeres de establecimientos con niveles bajos de exposición. Estar casada y tener una pareja fija actual también se mantuvieron asociadas de manera significativa con el uso inconsistente de condones. La ciudad donde labora también se mantuvo como un determinante significativo del UCC controlando por las otras variables del estudio, con la excepción del cumplimiento observado con los cinco componentes claves de la intervención. Sin embargo, cuando se controló por la variable del cumplimiento observado en el modelo, la asociación significativa entre ciudad y el UCC desapareció. 
Tabla 5 Efectos de la exposición a la intervención y el cumplimiento en el uso consistente de condones en el último mes, post-intervención ( $n=333$ )

\begin{tabular}{|c|c|c|c|c|c|c|}
\hline \multirow[t]{2}{*}{ Variables } & \multicolumn{3}{|c|}{ OR No-ajustada } & \multicolumn{3}{|c|}{ OR Ajustada } \\
\hline & OR & $95 \%$ IC & Valor $\mathbf{p}$ & OR & $95 \%$ IC & Valor $p$ \\
\hline \multicolumn{7}{|l|}{$\begin{array}{l}\text { Exposición reporta- } \\
\text { da a la intervención } \\
\text { Baja }\end{array}$} \\
\hline Alta & 1.99 & $1.28-3.08$ & 0.002 & 1.84 & $1.07-3.17$ & 0.027 \\
\hline \multicolumn{7}{|l|}{$\begin{array}{l}\text { Nivel promedio de } \\
\text { cumplimiento } \\
\text { observado con la } \\
\text { intervención }\end{array}$} \\
\hline $0.0-3.0$ & & & & & & \\
\hline $3.01-3.50$ & 2.28 & $1.22-4.30$ & 0.010 & 1.60 & $.79-3.25$ & 0.195 \\
\hline $3.51-4.00$ & 3.71 & $1.98-6.94$ & 0.000 & 2.43 & $.97-6.15$ & 0.059 \\
\hline$>4.00$ & 3.76 & $1.95-7.26$ & 0.000 & 2.33 & $1.01-5.39$ & 0.047 \\
\hline \multicolumn{7}{|l|}{ Ciudad } \\
\hline $\begin{array}{l}\text { Puerto Plata } \\
\text { Edad en años }\end{array}$ & 2.29 & $1.48-3.56$ & 0.000 & 1.45 & $.68-3.06$ & 0.332 \\
\hline $18-25$ & & & & & & \\
\hline $26+$ & .97 & $.62-1.50$ & 0.876 & .98 & $.60-1.60$ & 0.936 \\
\hline \multicolumn{7}{|l|}{ Educación en años } \\
\hline $\begin{array}{l}9+ \\
\text { Estado civil }\end{array}$ & .91 & $.56-1.48$ & 0.696 & .89 & $.45-1.76$ & 0.743 \\
\hline $\begin{array}{l}\text { Soltera/divorciada } \\
\text { Casada/en unión }\end{array}$ & .24 & $.15-.39$ & 0.000 & .40 & $.26-.61$ & 0.000 \\
\hline $\begin{array}{l}\text { Actualmente tiene } \\
\text { pareja fija } \\
\text { No }\end{array}$ & & & & & & \\
\hline $\begin{array}{l}\text { Si } \\
\text { Número de citas en } \\
\text { la semana pasada }\end{array}$ & .08 & $.04-.18$ & 0.000 & .10 & $.04-.25$ & 0.000 \\
\hline $\begin{array}{l}<2 \\
2+\end{array}$ & 1.68 & $1.10-2.60$ & 0.020 & 1.22 & $.63-2.35$ & 0.560 \\
\hline $\begin{array}{l}\text { Número total de } \\
\text { parejas en el último } \\
\text { mes }\end{array}$ & & & & & & \\
\hline$<3$ & & & & & & \\
\hline $\begin{array}{l}3+ \\
\text { Tarifa promedio } \\
\text { cobrada por cita }\end{array}$ & 1.07 & $.69-1.65$ & 0.761 & .68 & $.32-1.45$ & 0.321 \\
\hline US\$0-18 & & & & & & \\
\hline US\$19+ & 1.53 & $.98-2.40$ & 0.064 & 1.24 & $.72-2.13$ & 0.433 \\
\hline
\end{tabular}




\section{Hgrizons}

\section{Otros Resultados de la I ntervención}

Varios indicadores adicionales fueron evaluados en este estudio para examinar los efectos de la intervención en las percepciones de los participantes sobre su ambiente de trabajo y para asegurar que la intervención no tuvo ninguna consecuencia negativa no intencionada para las mujeres participantes.

Se evaluó el abuso o maltrato por dueños de establecimientos de sexo, empleados masculinos, y clientes masculinos en los últimos tres meses reportado por las trabajadoras sexuales participantes en el estudio. En el caso de Puerto Plata, no se encontró ningún cambio significativo en el abuso o maltrato por ninguno de los tres grupos entre la encuesta pre- y post-intervención. En Santo Domingo, un cambio positivo fue documentado: el nivel reportado de abuso o maltrato en los últimos tres por parte de clientes masculinos hacia trabajadoras sexuales bajó de la encuesta preintervención a la post de un 30.2 por ciento a un 18.0 por ciento $(\mathrm{p}<0.015)$. También se preguntó a las trabajadoras sexuales si los dueños de los establecimientos les habían impuesto algún tipo de multa en los últimos tres meses. Frecuentemente se ponen multas a las trabajadoras sexuales por llegar tarde al trabajo o por otras cosas no relacionadas a la intervención. El porcentaje de trabajadoras sexuales que reportaron haber recibido una multa por cualquier razón en los últimos tres meses no cambió de manera significativa en ninguna ciudad entre la encuesta pre- y postintervención.

El último taller con cada uno de los tres principales grupos de la intervención (trabajadoras sexuales, dueños/administradores y otros empleados masculinos de establecimientos) proveyó una oportunidad para discutir con los participantes sus experiencias en la intervención. Adicionalmente, se hicieron preguntas abiertas con las trabajadoras sexuales en la encuesta post-intervención de cómo la intervención puede haberlas afectado a ellas y en sus relaciones con los dueños/administradores, empleados, clientes y otras parejas de manera positiva o negativa. Los tres grupos de la intervención reportaron sobre elementos positivos y útiles de la intervención. Por ejemplo, muchas trabajadoras sexuales reportaron que la intervención les ayudó a valorarse a ellas mismas y protegerse a ella y a su familia. Empleados de los establecimientos reportaron que por primera vez fueron tomados en cuenta y tuvieron la oportunidad de contribuir de manera positiva a la sociedad. Adicionalmente, los tres grupos reportaron una conciencia más alta de los retos en la prevención de las ITS y VIH y documentaron la importancia de trabajar con este tema de manera colectiva y no aislada.

El último taller también facilitó una oportunidad para discutir las actitudes y reacciones de los dueños de los establecimientos hacia el modelo con la política regional gubernamental y el sistema de sanciones graduadas en Puerto Plata. Durante el curso de la intervención en Puerto Plata, se impusieron 113 notificaciones, 18 multas y una clausura temporal en los establecimientos participantes en la intervención en esta ciudad, por falta de cumplimiento con los cinco elementos claves de la intervención de manera mensual. En general, los dueños de Puerto Plata reportaron que cumplir con los cinco elementos de la intervención de manera mensual fue un reto. Sin embargo, la mayoría expresaron su preferencia por la política transparente y el sistema de regulación utilizado en la iniciativa de 100 por ciento condones, la cual había sido desarrollada con la participación e 
ideas de ellos y otras personas claves involucradas, comparado con el antiguo sistema de monitoreo y cumplimiento, el cual tuvo muchos reportes de corrupción y manipulación.

\section{Análisis de Costo-efectividad}

\section{Métodos}

El equipo de investigación construyó un modelo para medir el costo-efectividad de la implementación de una intervención ambiental-estructural en Santo Domingo y Puerto Plata. Los resultados del modelo incluyeron los costos del programa, el número de infecciones de VIH evitado, el costo por infección evitada y el costo por Año de Vida Ajustado por Discapacidad (AVAD) salvado por la intervención. Resultados fueron modelados sobre la base del impacto para 10,000 trabajadoras sexuales alcanzadas por la intervención en cada ciudad.

Los investigadores examinaron el costo de la intervención desde la perspectiva de las ONGs que la implementaron. Aunque el gobierno participó en la intervención con el diagnóstico y tratamiento de ITS y con la implementación de sanciones en Puerto Plata, los costos de los mismos fueron insignificantes ya que tanto los servicios de ITS como el monitoreo de los establecimientos de sexo ya estaban siendo proveídos por el gobierno. El mayor aporte adicional del gobierno fue en el monitoreo del cumplimiento con los elementos ambientales de la intervención y la implementación de sanciones, los cuales fueron realizados en el mismo momento de sus inspecciones rutinarias. Los inspectores gubernamentales acompañados por personal de las ONGs recolectaron los resultados de los chequeos mensuales de las TRSX en las clínicas públicas y en caso de una prueba positiva para ITS, los dueños de los establecimientos fueron sancionados. Así que, para este análisis la pregunta de investigación fue: “¿Cuál es el costo-efectividad de la implementación de cada modelo de intervención ambiental-estructural en áreas geográficas que ya cuentan con un sistema de chequeo mensual de ITS para trabajadoras sexuales?"

Estimación de los AVAD salvados. Para estimar el número de AVAD salvados por la intervención, calculamos el número de AVAD perdidos por una infección del VIH debido a la discapacidad asociada con infección del VIH además de la muerte prematura por SIDA. Se estimó la esperanza de vida en la edad promedia de infección con VIH sobre la base de una revisión de datos demográficos disponibles (United Nations Population Division 2001). Esta estimación está basada en una esperanza de vida independiente de la infección con VIH para el análisis del caso-base. Los pesos de la discapacidad por infección con VIH y SIDA fueron estimados en 0.123 y 0.505 , respectivamente (Murray and Lopez 1996). Parámetros de la progresión de la enfermedad fueron adaptados de la literatura científica publicada (Hendricks et al. 1996), donde se estima que son ocho años para el progreso desde la infección con VIH-1 hasta el SIDA y una progresión de un año desde el SIDA hasta la muerte, como fue recomendado por UNAIDS para países en desarrollo.

Se ajustó el número de AVAD salvados por edad y se descontó el número de AVAD salvados para reflejar preferencias por la edad utilizando técnicas analíticas estándares (Murray et al. 1996). Costos y beneficios futuros fueron descontados a una tasa del 3 por ciento, con un análisis de sensibilidad de 0 por ciento a 6 por ciento. El número total de AVAD salvado de la intervención 


\section{Hgrizons}

fue calculado multiplicando el número anual de infecciones evitadas por el número de AVAD de discapacidad y mortalidad temprana salvado por infección de VIH evitada.

Medición de los costos. Los costos de la intervención fueron calculados estimando la cantidad de insumos y servicios por cliente utilizados en la intervención. Estos cálculos fueron derivados de formularios de costos creados por el proyecto, los registros y presupuestos del proyecto y entrevistas con personal y gerentes del proyecto. Todos los costos de la investigación fueron eliminados y las estimaciones finales de costo de la intervención fueron ajustadas considerando el costo actual de la provisión de servicios cuando se finalizó la investigación y cada ciudad se dedicaba exclusivamente a la provisión de servicios. El costo por cliente de la intervención refleja el costo para la ONG que la implementó.

La participación del gobierno incurrió costos insignificantes porque los servicios de ITS ya estaban funcionando y los inspectores gubernamentales ya estaban visitando los establecimientos de sexo. Los resultados del análisis están presentados sobre la base de 10,000 clientes bajo la suposición de que la intervención fue implementada de manera completa en un lugar dado y también para permitir la comparación con otros análisis de costo-efectividad. Todos los costos fueron convertidos a dólares de EEUU en 2001 para su presentación.

Modelo del estudio. Estimaciones del número de infecciones de VIH evitadas fueron derivadas de la siguiente fórmula de común uso basada en la probabilidad (Weinstein et al. 1989):

[Ecuación 1]: Probabilidad de Infección con VIH (P) $=1-\left\{p[1-r(1-f e)]^{n}+(1-p)\right\}^{m}$

Donde $p$ es la prevalencia promedio de VIH en las parejas sexuales de la población meta (trabajadoras sexuales), $r$ es el riesgo de transmisión de VIH por acto sexual no protegido (también llamado "infectividad"), $f$ es la proporción de actos sexuales realizados con un condón, $e$ es la efectividad de los condones, $n$ es el número promedio de actos sexuales realizados con un condón, y m es el número promedio de parejas sexuales. Estimaciones para el modelo fueron calculados individualmente para cada ciudad y también por tipo de pareja (pareja fija que paga, pareja fija que no paga, y clientes nuevos que pagan). Dado que las trabajadoras sexuales que fueron la población meta para la intervención están en riesgo por dos tipos de parejas (clientes nuevos y parejas fijas), cada una incurriendo en una estimación distinta de la probabilidad de infectar una mujer, se combinó el riesgo anual de ambos tipos de parejas con la siguiente formula:

[Ecuación 2]: Probabilidad Combinada de Infección con VIH $=\left((\operatorname{Pr}+\operatorname{Pn})_{-}(\operatorname{Pr} * \operatorname{Pn})\right)$

Donde $\operatorname{Pr}$ es la probabilidad anual de infección con VIH de parejas fijas (que pagan y non pagan), y $P n$ es la probabilidad anual de infección con VIH de clientes nuevos que pagan. En la Ecuación 1 se calcula $\operatorname{Pr}$ y $\operatorname{Pn}$ separadas, y después combinadas en la Ecuación 2. Entonces, el modelo estima la transmisión de VIH desde tres fuentes que podrían ser afectados por la intervención: (a) trabajadoras sexuales VIH-negativas de la población meta de la intervención que se infectan por parejas fijas y clientes nuevos, (b) parejas fijas VIH-negativas que se infectan por trabajadoras sexuales de la población meta de la intervención, (c) clientes nuevos VIH-negativos que se infectan por trabajadoras sexuales de la población meta de la intervención. 
Análisis de sensibilidad. Se realizó un análisis de sensibilidad utilizando un modelo Monte Carlo para examinar como las variaciones en los parámetros epidemiológicos, económicos y de comportamiento del modelo afectan el costo-efectividad de la intervención. El modelo estocástico fue diseñado del modelo de caso-base utilizando funciones triangulares de la distribución de la probabilidad con valores alto, promedio y bajo. Muchos de los valores epidemiológicos y económicos fueron estimados de informes publicados. Datos de comportamiento e ITS fueron tomados de los datos del estudio y de las estimaciones de punto. Para el análisis de sensibilidad, se utilizaron los límites mayores y menores de los intervalos de confianza de 95 por ciento. Se realizó iteraciones del modelo hasta que los cambios en los valores de las variables y resultados asociados cambiaron por menos de 1.5 por ciento tanto en la media como en la desviación estándar.

El producto resultante es un conjunto de valores variables de los insumos del modelo (simultáneamente en todos los parámetros), con resultados asociados para cada iteración del modelo. Los grupos de parámetros fueron fijados o permitidos a variar en diferentes simulaciones del modelo para los análisis de sensibilidad de una, dos y tres vías. El modelo estocástico también fue usado para el análisis de sensibilidad multi-variable.

\section{Parámetros del modelo}

Epidemiológicos. Valores de caso-base para los parámetros del modelo epidemiológico y rangos asociados para cada parámetro utilizado en el análisis de sensibilidad están mostrados en la Tabla 6. La sero-prevalencia de VIH en trabajadoras sexuales fue estimada de estudios de vigilancia recientemente realizados en cada ciudad. Los investigadores suponían que la prevalencia de VIH en las parejas sexuales (clientes nuevos y parejas fijas) de trabajadoras sexuales VIH-positivas era igual a la prevalencia en la población de trabajadoras sexuales y que para las parejas sexuales de trabajadoras sexuales no-infectadas con VIH, la prevalencia era de un 25 por ciento menos. Los intervalos de confianza de 95 por ciento fueron utilizados en el análisis de sensibilidad. Los valores de la infectividad de VIH-1 son de datos publicados y fueron ajustados por co-infección con ITS. La infectividad de VIH-1 de hombre-a-mujer se supuso en un 0.002 por acto sexual entre un hombre infectado y una mujer no infectada; para la infectividad de mujer-a-hombre, la suposición fue 0.001 por acto sexual para mujer infectada a hombre no infectado (Rehle et al. 1998). En caso de una ITS ulcerativa en cualquier persona de la pareja, la tasa de transmisión fue supuesta en 0.06; con una ITS no ulcerativa (Clamidia, Tricomoniasis, y gonorrea), la tasa fue supuesta en 0.02 de hombre-a-mujer y 0.01 de mujer-a-hombre (Rehle et al. 1998).

La prevalencia de ITS no ulcerativas fue observada en el estudio, y se utilizaron las tasas de las encuestas pre- y post-intervención en el modelo para estimar el promedio ajustado de la infectividad con VIH. Las tasas de ITS genitales ulcerativas fueron estimadas en un 5 por ciento para las trabajadoras sexuales. Ya que las ITS ulcerativas no fueron evaluadas en trabajadoras sexuales en este estudio, se optó por establecer valores conservativos como una constante desde la encuesta pre-intervención hasta la encuesta post-intervención. Los valores pre- y post-intervención para la tasa de infectividad de VIH ajustada están mostrados en la Tabla 6 por hombres y mujeres por ciudad. 
Tabla 6 Valores de caso-base para parámetros epidemiológicos (periodo de 1 año)

\begin{tabular}{|c|c|c|c|c|}
\hline Parámetros & Ciudad & $\begin{array}{l}\text { Estimación } \\
\text { Caso-Base }\end{array}$ & Rango & $\begin{array}{l}\text { Fuente de } \\
\text { datos }\end{array}$ \\
\hline \multirow{2}{*}{$\begin{array}{l}\text { Prevalencia de VIH en } \\
\text { trabajadoras sexuales } \\
\text { (TRSX) }\end{array}$} & Puerto Plata & & & \multirow{4}{*}{$\begin{array}{l}\text { Vigilancia } \\
\text { centinela }(95 \% \\
\text { IC) }\end{array}$} \\
\hline & Santo Domingo & & & \\
\hline \multirow{2}{*}{$\begin{array}{l}\text { Prevalencia de VIH en } \\
\text { clientes nuevos (CN) y } \\
\text { parejas fijas (PF) de } \\
\text { TRSX VIH+ }\end{array}$} & Puerto Plata & $5.68 \%$ & $(3.38-7.99 \%)$ & \\
\hline & Santo Domingo & & & \\
\hline Prevalencia de VIH en CN & Puerto Plata & & & \multirow{2}{*}{$\begin{array}{l}\text { Estimado } 25 \% \\
\text { menos que prev. } \\
\text { en TRSX }\end{array}$} \\
\hline $\begin{array}{l}\text { y PF de TRSX no- } \\
\text { infectadas con VIH }\end{array}$ & & $4.3 \%$ & $(2.54-5.99 \%)$ & \\
\hline \multirow{2}{*}{$\begin{array}{l}\text { Prevalencia de ITS no } \\
\text { ulcerativas pre- } \\
\text { intervención }\end{array}$} & Puerto Plata & $28.8 \%$ & $22.4-35.2 \%$ & \multirow{4}{*}{$\begin{array}{l}\text { Observado } \\
\text { (Rango 95\% IC) }\end{array}$} \\
\hline & Santo Domingo & $25.5 \%$ & $19.7-31.3 \%$ & \\
\hline \multirow{2}{*}{$\begin{array}{l}\text { Prevalencia de ITS no } \\
\text { ulcerativas post- } \\
\text { intervención }\end{array}$} & Puerto Plata & $16.3 \%$ & $11.1-21.4 \%$ & \\
\hline & Santo Domingo & $15.9 \%$ & $11.0-20.8 \%$ & \\
\hline \multirow{2}{*}{$\begin{array}{l}\text { Estimación pre/post de } \\
\text { prevalencia de ITS } \\
\text { ulcerativas }\end{array}$} & Puerto Plata & $5 \%$ & $(2 \%-10 \%)$ & \multirow{2}{*}{$\begin{array}{l}\text { Estimado de } \\
\text { datos de } \\
\text { vigilancia }\end{array}$} \\
\hline & Santo Domingo & $5 \%$ & $(2 \%-10 \%)$ & \\
\hline \multirow{2}{*}{$\begin{array}{l}\text { Probabilidad de infección } \\
\text { con VIH por acto sexual } \\
\text { en TRSX no infectadas } \\
\text { con VIH pre-intervención }\end{array}$} & Puerto Plata & 0.00559 & $\begin{array}{c}0.00278- \\
0.00834\end{array}$ & \multirow{8}{*}{$\begin{array}{l}\text { Tasa base de } \\
0.001 \text { ajustado } \\
\text { por co-infección } \\
\text { con ITS } \\
\text { (este rango } \\
\text { varía con el } \\
\text { rango de ITS } \\
\text { ajustado) }\end{array}$} \\
\hline & Santo Domingo & 0.00541 & $\begin{array}{c}0.00271- \\
0.00812\end{array}$ & \\
\hline \multirow{2}{*}{$\begin{array}{l}\text { Probabilidad de infección } \\
\text { con VIH por acto sexual } \\
\text { en TRSX no infectadas } \\
\text { con VIH post-intervención }\end{array}$} & Puerto Plata & 0.00496 & $\begin{array}{l}0.00249 \\
0.00744\end{array}$ & \\
\hline & Santo Domingo & 0.00482 & $\begin{array}{c}0.00241- \\
0.00723\end{array}$ & \\
\hline \multirow{2}{*}{$\begin{array}{l}\text { Probabilidad de infección } \\
\text { con VIH por acto sexual } \\
\text { en CN y PF no infectadas } \\
\text { con VIH pre-intervención }\end{array}$} & Puerto Plata & 0.00642 & $\begin{array}{c}0.00321- \\
0.00963\end{array}$ & \\
\hline & Santo Domingo & 0.00633 & $\begin{array}{c}0.00317- \\
0.00950\end{array}$ & \\
\hline \multirow{2}{*}{$\begin{array}{l}\text { Probabilidad de infección } \\
\text { con VIH por acto sexual } \\
\text { en CN y PF no infectadas } \\
\text { con VIH post-intervención }\end{array}$} & Puerto Plata & 0.00579 & $\begin{array}{c}0.00290- \\
0.00869\end{array}$ & \\
\hline & Santo Domingo & 0.00574 & $\begin{array}{c}0.00287- \\
0.00861\end{array}$ & \\
\hline
\end{tabular}


De comportamiento. Los parámetros de comportamiento sexual fueron estimados de preguntas específicas a los participantes en el estudio con relación al número y tipo de parejas sexuales, número y tipo de actos sexuales y el uso consistente de condones por tipo de pareja en el último mes (ver página 15 para las definiciones completas de estos parámetros). Todos los valores de comportamiento fueron estandarizados para un año. El análisis de sensibilidad fue realizado en estos parámetros de comportamiento sexual utilizando los límites altos y bajos de los intervalos de confianza del 95 por ciento. Algunos parámetros para las parejas sexuales de trabajadoras sexuales fueron estimados, como se indica en la Tabla 7. Se optó por usar los datos de comportamiento de auto-reporte de trabajadoras sexuales, en lugar de los datos de las observaciones participativas. Esta es una estrategia más conservadora (porque es más parcial hacia los resultados menos costoefectivo) que proveyó una muestra más grande para el análisis y permitió la generación de estimaciones por tipo de pareja. 
Tabla 7 Valores de caso-base para parámetros de comportamiento (periodo 1-año)

\begin{tabular}{|c|c|c|c|c|}
\hline Parámetro & Ciudad & $\begin{array}{l}\text { Estimación } \\
\text { caso-base }\end{array}$ & $95 \%$ IC & Fuente de datos \\
\hline \multicolumn{5}{|l|}{$\begin{array}{l}\text { Para parejas fijas } \\
\text { (que pagan y que no pagan) }\end{array}$} \\
\hline \multirow{2}{*}{$\begin{array}{l}\text { Porcentaje de uso } \\
\text { consistente de condón } \\
\text { (UCC) pre-intervención }\end{array}$} & Puerto Plata & $13.0 \%$ & $7.2-18.1 \%$ & Observado (95\% IC) \\
\hline & Santo Domingo & $14.6 \%$ & $8.8-20.4 \%$ & Observado (95\% IC) \\
\hline \multirow{2}{*}{$\begin{array}{l}\text { Porcentaje de UCC post- } \\
\text { intervención }\end{array}$} & Puerto Plata & $28.8 \%$ & $21.5-36.4 \%$ & Observado (95\% IC) \\
\hline & Santo Domingo & $17.6 \%$ & $11.7-23.5 \%$ & Observado (95\% IC) \\
\hline \multirow{2}{*}{$\begin{array}{l}\text { Número promedio de actos } \\
\text { sexuales por pareja pre- } \\
\text { intervención }\end{array}$} & Puerto Plata & 50.7 & 24-80 & Estimado \\
\hline & Santo Domingo & 41.7 & $20-64$ & Estimado \\
\hline \multirow{2}{*}{$\begin{array}{l}\text { Número promedio de } \\
\text { parejas sexuales en parejas } \\
\text { fijas (pre- y post- } \\
\text { intervención) }\end{array}$} & Puerto Plata & 3 & $2-4$ & Estimado \\
\hline & Santo Domingo & 3 & $2-4$ & Estimado \\
\hline \multirow{2}{*}{$\begin{array}{l}\text { Número promedio de } \\
\text { parejas sexuales en TRSX } \\
\text { (pre- y post- } \\
\text { intervención) }\end{array}$} & Puerto Plata & 5 & $2.8-7.2$ & Observado (95\% IC) \\
\hline & Santo Domingo & 3 & $1.7-4.3$ & Observado (95\% IC) \\
\hline \multicolumn{5}{|l|}{ Para clientes nuevos que pagan } \\
\hline \multirow{2}{*}{$\begin{array}{l}\text { Porcentaje de UCC pre- } \\
\text { intervención }\end{array}$} & Puerto Plata & $96.5 \%$ & $93.1-99.9 \%$ & Observado (95\% IC) \\
\hline & Santo Domingo & $75.3 \%$ & $66.3-84.2 \%$ & Observado (95\% IC) \\
\hline \multirow{2}{*}{$\begin{array}{l}\text { Porcentaje de UCC post- } \\
\text { intervención }\end{array}$} & Puerto Plata & $98.6 \%$ & $96.6-99.9 \%$ & Observado (95\% IC) \\
\hline & Santo Domingo & $93.8 \%$ & $89.6-98.3 \%$ & Observado (95\% IC) \\
\hline \multirow{2}{*}{$\begin{array}{l}\text { Número promedio de actos } \\
\text { sexuales por pareja pre- } \\
\text { intervención }\end{array}$} & Puerto Plata & 2 & $1-3$ & Estimado \\
\hline & Santo Domingo & 2 & $1-3$ & Estimado \\
\hline \multirow[t]{2}{*}{$\begin{array}{l}\text { Número promedio de actos } \\
\text { sexuales en clientes nuevos } \\
\text { (pre- y post-intervención) }\end{array}$} & Puerto Plata & 11.4 & $4.9-19.5$ & $\begin{array}{l}\text { Estimado: bajo } 12.5 \% \\
\text { de mujeres (39), med } \\
25 \% \text {, y alto } 50 \%\end{array}$ \\
\hline & Santo Domingo & 5.88 & $2.5-10$ & $\begin{array}{l}\text { Estimado: bajo } 12.5 \% \\
\text { de mujeres }(20), \text { med } \\
25 \% \text {, y alto } 50 \%\end{array}$ \\
\hline \multirow{2}{*}{$\begin{array}{l}\text { Número promedio de } \\
\text { parejas sexuales en TRSX } \\
\text { (pre- y post-intervención) }\end{array}$} & Puerto Plata & 39 & $22-56$ & Observado (95\% IC) \\
\hline & Santo Domingo & 20 & $11-29$ & Observado (95\% IC) \\
\hline
\end{tabular}


Económico. El costo por cliente para implementar la intervención ambiental-estructural y el porcentaje asociado de costos totales están mostrados en la Tabla 8. Por el hecho del diseño, el modelo de intervención fue muy parecido en ambas ciudades y las dos utilizaron presupuestos similares. Sin embargo, en Puerto Plata las autoridades gubernamentales de salud reforzaron su estrategia regulatoria utilizando recursos existentes, como fue descrito anteriormente. Dado que este análisis fue realizado desde la perspectiva de las ONGs que implementaron la intervención, los costos no varían mucho entre ciudades. Estos datos de costos vienen de los formularios de costo detallados que fueron llenados por gerentes en cada ciudad y por medio de consultas continuas con gerentes de las ONGs. Los formularios de costo fueron comparados con los presupuestos y fueron revisado y discutido con miembros del equipo del proyecto para refinar las estimaciones y para asegurar que ningún costo relacionado con la investigación fuese incluido. Las estimaciones de costo detalladas fueron agrupadas en las categorías mostradas, y los rangos de 25 por ciento por encima y por debajo de cada categoría de costo fueron incluidos para el análisis de sensibilidad. 
Tabla 8 Valores de caso-base para los parámetros económicos por TRSX por año

\begin{tabular}{|c|c|c|c|c|c|}
\hline Parámetro & Ciudad & $\begin{array}{l}\text { Estimación } \\
\text { Caso-Base }\end{array}$ & $\begin{array}{l}\text { Porcentaje } \\
\text { del costo } \\
\text { total }\end{array}$ & $\begin{array}{c}\text { Rango de } \\
\text { Estimaciones }\end{array}$ & Fuente de datos \\
\hline \multirow{2}{*}{$\begin{array}{l}\text { Costos } \\
\text { administrativos } \\
\text { y de } \\
\text { infraestructura }\end{array}$} & $\begin{array}{l}\text { Puerto } \\
\text { Plata }\end{array}$ & $\$ 14.38$ & 9.0 & $\$ 10.78-\$ 17.97$ & $\begin{array}{l}\text { Observado } \\
\text { (Rango +/- 25\%) }\end{array}$ \\
\hline & $\begin{array}{l}\text { Santo } \\
\text { Domingo }\end{array}$ & $\$ 14.38$ & 8.8 & $\$ 10.78-\$ 17.97$ & $\begin{array}{l}\text { Observado } \\
\text { (Rango }+/-25 \% \text { ) }\end{array}$ \\
\hline \multirow[t]{2}{*}{$\begin{array}{l}\text { Costos de } \\
\text { capacitación }\end{array}$} & $\begin{array}{l}\text { Puerto } \\
\text { Plata }\end{array}$ & $\$ 5.88$ & 3.7 & $\$ 4.41-\$ 7.35$ & $\begin{array}{l}\text { Observado } \\
\text { (Rango +/- 25\%) }\end{array}$ \\
\hline & $\begin{array}{c}\text { Santo } \\
\text { Domingo }\end{array}$ & $\$ 5.88$ & 3.6 & $\$ 4.41-\$ 7.35$ & $\begin{array}{l}\text { Observado } \\
\text { (Rango }+/-25 \% \text { ) }\end{array}$ \\
\hline \multirow[t]{2}{*}{ Personal } & $\begin{array}{l}\text { Puerto } \\
\text { Plata }\end{array}$ & $\$ 96.23$ & 60.3 & $\$ 72.18-\$ 120.29$ & $\begin{array}{l}\text { Observado } \\
\text { (Rango +/- 25\%) }\end{array}$ \\
\hline & $\begin{array}{l}\text { Santo } \\
\text { Domingo }\end{array}$ & $\$ 100.85$ & 61.4 & $\$ 75.64-\$ 126.06$ & $\begin{array}{l}\text { Observado } \\
\text { (Rango }+/-25 \% \text { ) }\end{array}$ \\
\hline \multirow[t]{2}{*}{$\begin{array}{l}\text { Talleres y } \\
\text { teatro }\end{array}$} & $\begin{array}{l}\text { Puerto } \\
\text { Plata }\end{array}$ & $\$ 18.15$ & 11.6 & $\$ 13.61-\$ 22.69$ & $\begin{array}{l}\text { Observado } \\
\text { (Rango }+/-25 \% \text { ) }\end{array}$ \\
\hline & $\begin{array}{l}\text { Santo } \\
\text { Domingo }\end{array}$ & $\$ 18.15$ & 11.1 & $\$ 13.61-\$ 22.69$ & $\begin{array}{l}\text { Observado } \\
\text { (Rango }+/-25 \%)\end{array}$ \\
\hline \multirow{2}{*}{$\begin{array}{l}\text { Insumos } \\
\text { básicos } \\
\text { (condones y } \\
\text { materiales } \\
\text { educativos) }\end{array}$} & $\begin{array}{l}\text { Puerto } \\
\text { Plata }\end{array}$ & $\$ 24.91$ & 15.6 & $\$ 18.68-\$ 31.13$ & $\begin{array}{l}\text { Observado } \\
\text { (Rango }+/-25 \% \text { ) }\end{array}$ \\
\hline & $\begin{array}{l}\text { Santo } \\
\text { Domingo }\end{array}$ & $\$ 24.91$ & 15.2 & $\$ 18.68-\$ 31.13$ & $\begin{array}{l}\text { Observado } \\
\text { (Rango +/- 25\%) }\end{array}$ \\
\hline \multirow[t]{2}{*}{$\begin{array}{l}\text { Costo total por } \\
\text { cliente }\end{array}$} & $\begin{array}{l}\text { Puerto } \\
\text { Plata }\end{array}$ & $\$ 159.55$ & 100.0 & $\$ 119.66-\$ 199.94$ & $\begin{array}{l}\text { Suma de los } \\
\text { valores arriba }\end{array}$ \\
\hline & $\begin{array}{l}\text { Santo } \\
\text { Domingo }\end{array}$ & $\$ 164.17$ & 100.0 & $\$ 123.13-\$ 205.21$ & $\begin{array}{l}\text { Suma de los } \\
\text { valores arriba }\end{array}$ \\
\hline
\end{tabular}




\section{Resultados}

\section{Resultados de caso-base}

La Tabla 9 demuestra los resultados de costo-efectividad del caso-base con relación al dólar de Estados Unidos en el año 2001 (redondeando el dólar) y están presentados suponiendo un programa que alcanzaría a 10,000 clientes por ciudad. En Puerto Plata, con las regulaciones gubernamentales reforzadas, se estima que la intervención evitaría 162 infecciones de VIH en 10,000 clientes alcanzados: 142 en trabajadoras sexuales, dos en clientes nuevos, y 19 en parejas fijas. En contraste, en Santo Domingo, se estima que la intervención evitaría 64 infecciones de VIH: 54 en trabajadoras sexuales, dos en clientes nuevos, y ocho en parejas fijas.

Vale la pena destacar que la intervención tiene su mayor impacto en la transmisión de VIH a trabajadoras sexuales. En los hombres, son las parejas fijas de trabajadoras sexuales, más que los clientes nuevos, quienes tienen la mayor posibilidad de beneficiarse por esta intervención. Además, el modelo de intervención implementado en Puerto Plata que incluyó la política gubernamental y regulación evitó 2.5 veces más infecciones que el modelo implementado en Santo Domingo. Como resultado, el costo por infección de VIH evitada en Puerto Plata, de \$9,843, es significativamente menor que en Santo Domingo, de \$25,574. Así, el costo por AVAD salvado es \$414 en Puerto Plata contra \$1,075 en Santo Domingo.

\section{Tabla 9 Costo-efectividad caso-base 1 año de la intervención 100\% condón por} 10,000 clientes

\begin{tabular}{|c|c|c|}
\hline & Puerto Plata & Santo Domingo \\
\hline \multirow{2}{*}{$\begin{array}{l}\text { Infecciones de VIH } \\
\text { evitadas }\end{array}$} & 162 & 64 \\
\hline & $\begin{array}{l}\text { - } 141 \text { en trabajadoras sexuales } \\
\text { - } 2 \text { en clientes nuevos que pagan } \\
\text { - } 19 \text { en parejas fijas (que pagan y } \\
\text { que no pagan) }\end{array}$ & $\begin{array}{l}\text { - } 54 \text { trabajadoras sexuales } \\
\text { - } 2 \text { en clientes nuevos que } \\
\text { pagan } \\
\text { - } 8 \text { en parejas fijas (que pagan y } \\
\text { que no pagan) }\end{array}$ \\
\hline $\begin{array}{l}\text { Costo por infección } \\
\text { de VIH evitada }\end{array}$ & $\$ 9,843$ & $\$ 25,574$ \\
\hline $\begin{array}{l}\text { Costo por AVAD } \\
\text { salvado }\end{array}$ & $\$ 414$ & $\$ 1,075$ \\
\hline
\end{tabular}




\section{Hgrizons}

\section{Análisis de sensibilidad: Análisis de una vía}

La Tabla 10 demuestra los resultados del análisis de sensibilidad de una vía, indicando el impacto de la variación de los parámetros individuales del modelo en el costo por AVAD salvado en cada ciudad. Tanto en Puerto Plata como en Santo Domingo el costo por AVAD salvado es más sensible a la tasa de descuento, la prevalencia de VIH, la estimación de AVAD salvado por infección de VIH evitada y la tasa de contacto entre trabajadoras sexuales y parejas fijas. La tasa de descuento es un ajuste hecho en el análisis para dar menos peso a los beneficios de salud futuros que actuales para las inversiones en salud. Por ejemplo, si se da el mismo valor a los beneficios a largo plazo que a los beneficios a corto plazo (ej. una tasa de descuento de 0 por ciento), se reduce el costo por AVAD salvado en Puerto Plata del valor del caso-base de US \$414 a US \$178.

La tasa de descuento de 3 por ciento utilizada en el análisis del caso-base es un estándar utilizado frecuentemente en análisis de costo-efectividad. Se demuestra que el costo-efectividad de la intervención 100 por ciento condones tiene una fuerte relación con la prevalencia de VIH en la población meta y sus parejas sexuales, y un efecto en el costo por AVAD salvado por los cambios en el número de años de vida salvados con cada infección evitada. También se encontró en el análisis de una vía que el costo-efectividad de la intervención fue sensible al número de parejas fijas que tiene cada trabajadora sexual, además de la frecuencia del contacto sexual con las parejas fijas. 
Tabla 10 Análisis de sensibilidad de una vía

Análisis de sensibilidad de una vía
Costo promedio por

AVAD salvado (\$USD / 2001)

Puerto

Plata

$\$ 414$

Caso-base (ver tablas anteriores para los valores y rangos de casobase)

Tasa de descuento

0\% Descontado

6\% Descontado

Infectividad de VIH (infectividad ajustada por género y prevalencia de ITS)

Número de parejas fijas (que pagan y no pagan)

Número de actos sexuales con parejas fijas (que pagan y no pagan)

Uso consistente de condones con parejas fijas (que pagan y no pagan)

Número de clientes nuevos que pagan

Número de actos sexuales con clientes nuevos que pagan

Uso consistente de condones con clientes nuevos que pagan

33\% Disminución de caso-base

$33 \%$ Aumento de caso-base

Máximo nivel del IC 95\% observado Mínimo nivel del IC 95\% observado

Máximo nivel del IC 95\% observado

Mínimo nivel del IC 95\% observado

Máximo nivel del IC 95\% observado

Mínimo nivel del IC 95\% observado

Máximo nivel del IC 95\% observado

Mínimo nivel del IC 95\% observado

Máximo nivel del IC 95\% observado

Mínimo nivel del IC 95\% observado

Máximo nivel del IC 95\% observado

Mínimo nivel del IC 95\% observado

$33 \%$ Aumento del caso-base

33\% Reducción del caso-base

33\% Aumento del caso-base

33\% Reducción del caso-base

$33 \%$ Aumento del caso-base

$33 \%$ Reducción del caso-base
$\$ 178$

$\$ 463$

$\$ 854$

$\$ 2,219$

$\$ 578$

$\$ 334$

$\$ 1,532$

$\$ 846$

$\$ 289$

$\$ 807$

$\$ 713$

$\$ 1,560$

$\$ 309$

$\$ 850$

$\$ 727$

$\$ 1,577$

$\$ 498$

$\$ 1,408$

$\$ 355$

$\$ 872$

$\$ 384$

$\$ 814$

$\$ 427$

$\$ 1,279$

$\$ 402$

$\$ 931$

$\$ 426$

$\$ 1,273$

$\$ 434$

$\$ 1,238$

$\$ 396$

$\$ 950$

$\$ 290$

$\$ 737$

$\$ 708$

$\$ 1,886$

$\$ 550$

$\$ 1,430$

$\$ 277$

$\$ 720$

$\$ 311$

$\$ 809$

$\$ 618$
$\$ 1,605$ 


\section{Hgrizons}

\section{Análisis de sensibilidad: Análisis multi-vía}

Se presentan los escenarios peores y mejores, donde todos los parámetros del modelo están fijados para el resultado más y menos costo-efectivo, respectivamente (Tabla 11; Figuras 2 y 3). El mejor y peor escenario también comunica el nivel de incertidumbre en el modelo.

En el peor de los casos, el costo por AVAD salvado va desde $\$ 414$ en Puerto Plata y $\$ 1,025$ en Santo Domingo, y de US \$1,194 y US \$6,202, respectivamente. En el mejor de los casos, el costo por AVAD salvado se reduce a US \$139 en Puerto Plata y US \$264 en Santo Domingo. Se examinó la sensibilidad de combinaciones de varios de los parámetros importantes en el modelo del análisis de una vía y se encontró que la combinación de la tasa de descuento y la infectividad de VIH, ambos parámetros basados en estimaciones de la literatura científica, explican la mayoría de la variabilidad en las estimaciones, como es el caso en muchos modelos de este tipo. En el análisis de tres vías, se ve otra vez que los parámetros económicos y epidemiológicos en combinación afectan los resultados del modelo mucho más que los datos de comportamiento que fueron recolectados directamente del estudio y que tienen mucho menos incertidumbre dado la oportunidad de generar intervalos de confianza del 95 por ciento para usar en el análisis de sensibilidad. Finalmente, se realizó un análisis de sensibilidad multi-variable regresando los resultados de las interacciones del modelo estocástico. Este análisis tiene especial valor porque en muchas ocasiones los parámetros del modelo co-varían, y el análisis multi-variable demuestra el efecto independiente de los parámetros del modelo con el resultado.

Los resultados de Puerto Plata y Santo Domingo son parecidos, siendo los parámetros más sensibles del modelo la tasa de descuento y la prevalencia de VIH en ambas ciudades. En Santo Domingo, la tasa de ITS también tuvo un fuerte efecto independiente en el costo por AVAD salvado. Adicionalmente, en ambas ciudades hay efectos fuertes de las tasas de contacto sexual con parejas fijas pre-intervención, especialmente cuando la pareja es VIH-positiva. Es interesante destacar que la variación en el costo de la intervención solo tiene un impacto moderado en el costoefectividad de la intervención, comparado con los parámetros epidemiológicos y de comportamiento del modelo. 
Tabla 11 Análisis de sensibilidad multi-vía

\begin{tabular}{|c|c|c|c|}
\hline & & \multicolumn{2}{|c|}{$\begin{array}{l}\text { Costo promedio por AVAD } \\
\text { salvado (\$USD / 2001) }\end{array}$} \\
\hline & & $\begin{array}{l}\text { Puerto } \\
\text { Plata }\end{array}$ & $\begin{array}{l}\text { Santo } \\
\text { Domingo }\end{array}$ \\
\hline \multicolumn{2}{|l|}{ Caso-base } & $\$ 414$ & $\$ 1,075$ \\
\hline \multicolumn{2}{|c|}{$\begin{array}{l}\text { Escenario del peor caso (todos los parámetros } \\
\text { establecidos en los valores del peor caso) }\end{array}$} & $\$ 1,194$ & $\$ 6,202$ \\
\hline \multicolumn{2}{|c|}{$\begin{array}{l}\text { Escenario del mejor caso (todos los parámetros } \\
\text { establecidos en los valores del mejor caso) }\end{array}$} & $\$ 139$ & $\$ 264$ \\
\hline \multicolumn{4}{|c|}{ Análisis de sensibilidad de dos vías } \\
\hline \multirow[t]{2}{*}{$\begin{array}{l}\text { (1) Tasa de descuento por } \\
\text { (2) Infectividad de VIH }\end{array}$} & $\begin{array}{l}\text { Peor caso (alto } \\
\text { descuento/baja infectividad) }\end{array}$ & $\$ 1,133$ & $\$ 3,423$ \\
\hline & $\begin{array}{l}\text { Mejor caso (bajo } \\
\text { descuento/alta infectividad) }\end{array}$ & $\$ 251$ & $\$ 533$ \\
\hline \multirow{2}{*}{$\begin{array}{l}\text { (1) Número de parejas } \\
\text { fijas que pagan y no } \\
\text { pagan (PF) por (2) uso } \\
\text { consistente de condones } \\
\text { (UCC) con PF post- } \\
\text { intervención }\end{array}$} & $\begin{array}{l}\text { Peor caso (bajo \# PF/bajo } \\
\text { UCC) }\end{array}$ & $\$ 719$ & $\$ 1,552$ \\
\hline & $\begin{array}{l}\text { Mejor caso (alto \# PF/alto } \\
\text { UCC) }\end{array}$ & $\$ 283$ & $\$ 819$ \\
\hline \multirow{2}{*}{$\begin{array}{l}\text { (1) Prevalencia VIH por (2) } \\
\text { número de clientes } \\
\text { nuevos que pagan }(\mathrm{CN})\end{array}$} & $\begin{array}{l}\text { Peor caso (baja } \\
\text { prevalencia/bajo \# CN) }\end{array}$ & $\$ 694$ & $\$ 1,838$ \\
\hline & $\begin{array}{l}\text { Mejor caso (alta } \\
\text { prevalencia/bajo \# CN) }\end{array}$ & $\$ 287$ & $\$ 740$ \\
\hline \multicolumn{4}{|l|}{ Análisis de tres vías } \\
\hline $\begin{array}{l}\text { Económico y } \\
\text { epidemiológico: }\end{array}$ & $\begin{array}{l}\text { Peor caso (alto costo/bajo } \\
\text { AVAD/baja infectividad) }\end{array}$ & $\$ 1,498$ & $\$ 3,998$ \\
\hline $\begin{array}{l}\text { (1) costo de la } \\
\text { intervención por (2) AVAD } \\
\text { por infección de VIH } \\
\text { evitada por (3) infectividad } \\
\text { de VIH }\end{array}$ & $\begin{array}{l}\text { Mejor caso(bajo costo/alto } \\
\text { AVAD/alta infectividad) }\end{array}$ & $\$ 133$ & $\$ 345$ \\
\hline \multirow{2}{*}{$\begin{array}{l}\text { De comportamiento: (1) } \\
\text { porcentaje de UCC (2) } \\
\text { número de PF (3) número } \\
\text { de actos sexuales }\end{array}$} & $\begin{array}{l}\text { Peor caso (bajo UCC/bajo \# } \\
\text { PF/bajo \# actos sexuales) }\end{array}$ & $\$ 1,037$ & $\$ 1,922$ \\
\hline & $\begin{array}{l}\text { Mejor caso (alto UCC/alto \# } \\
\text { PF/alto \# actos sexuales }\end{array}$ & $\$ 234$ & $\$ 633$ \\
\hline
\end{tabular}


Tabla 11 (continuada) Análisis de sensibilidad multi-variable - Modelo de regresión Asociación entre parámetros del modelo y costo promedio por AVAD salvado. (Coeficientes beta estandardizado mayor que 0.10 mostrados)

Santo Domingo. $\mathrm{R}^{2}=0.83$. (1) Tasa de descuento [0.56], (2) Prevalencia de VIH [-0.34], (3) ITS no ulcerativa en TRSX pre-intervención [-0.33], (4) ITS no ulcerativa en TRSX post-intervención [0.29], (5) Número de actos sexuales con parejas fijas en TRSX VIH + pre-intervención [-0.21], (6) Uso consistente de condón con parejas fijas en TRSX VIH+ pre-intervención [0.20], (7) Uso consistente de condón con parejas fijas de TRSX VIH + post-intervención [-0.18], (8) Número de parejas fijas en TRSX VIH- preintervención [-0.17], (9) Costos de personal [0.11], (10) Número de actos sexuales con clientes nuevos en TRSX VIH+ pre-intervención [-0.11], (11) Uso consistente de condón con clientes nuevos en TRSX VIH + pre-intervención [0.10].

Puerto Plata. $R^{2}=0.89$. (1) Tasa de descuento [0.64], (2) Prevalencia de VIH [-0.38], (3) Número de actos sexuales con parejas fijas en TRSX VIH+ pre-intervención [-0.35], (4) Número de parejas fijas preintervención en TRSX VIH+ [-0.30], (5) ITS no ulcerativa en TRSX pre-intervención [-0.24], (6) ITS no ulcerativa en TRSX post-intervención [0.19], (7) Uso consistente de condón con parejas fijas en TRSX $\mathrm{VIH}+$ pre-intervención [0.15], (8) Uso consistente de condón con parejas fijas de TRSX VIH + postintervención [-0.15], (9) Costos de personal [0.12].

\section{Figura 2 Regresión del análisis de sensibilidad: Puerto Plata}

Tasa de descuento

Prevalencia de VIH

\# actos sex. con PF en TRSX VIH+ pre-interv.

\# de PF en TRSX VIH+ pre-interv.

ITS no ulcerativa en TRSX pre-interv.

ITS no ulcerativa en TRSX post-interv.

UCC con PF en TRSX VIH+ pre-interv.

UCC con PF en TRSX VIH+ post-interv.

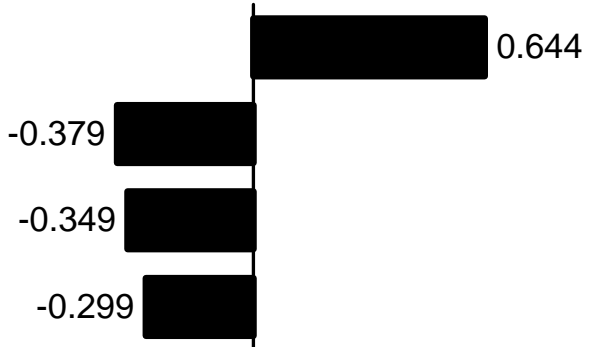

$-0.237$

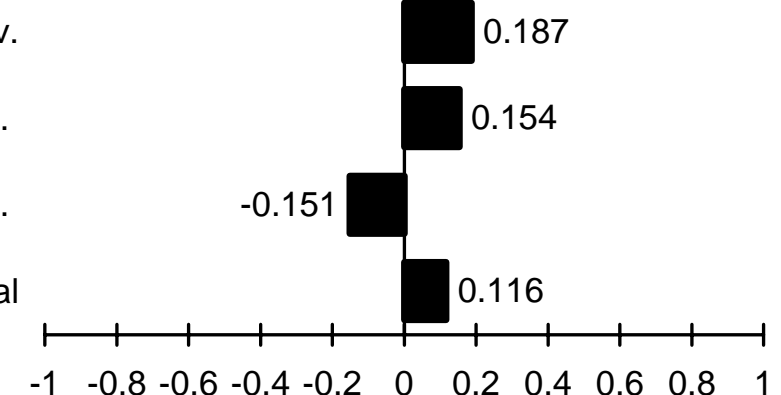

Coeficientes est. b 
Figura 3 Regresión del análisis de sensibilidad: Santo Domingo

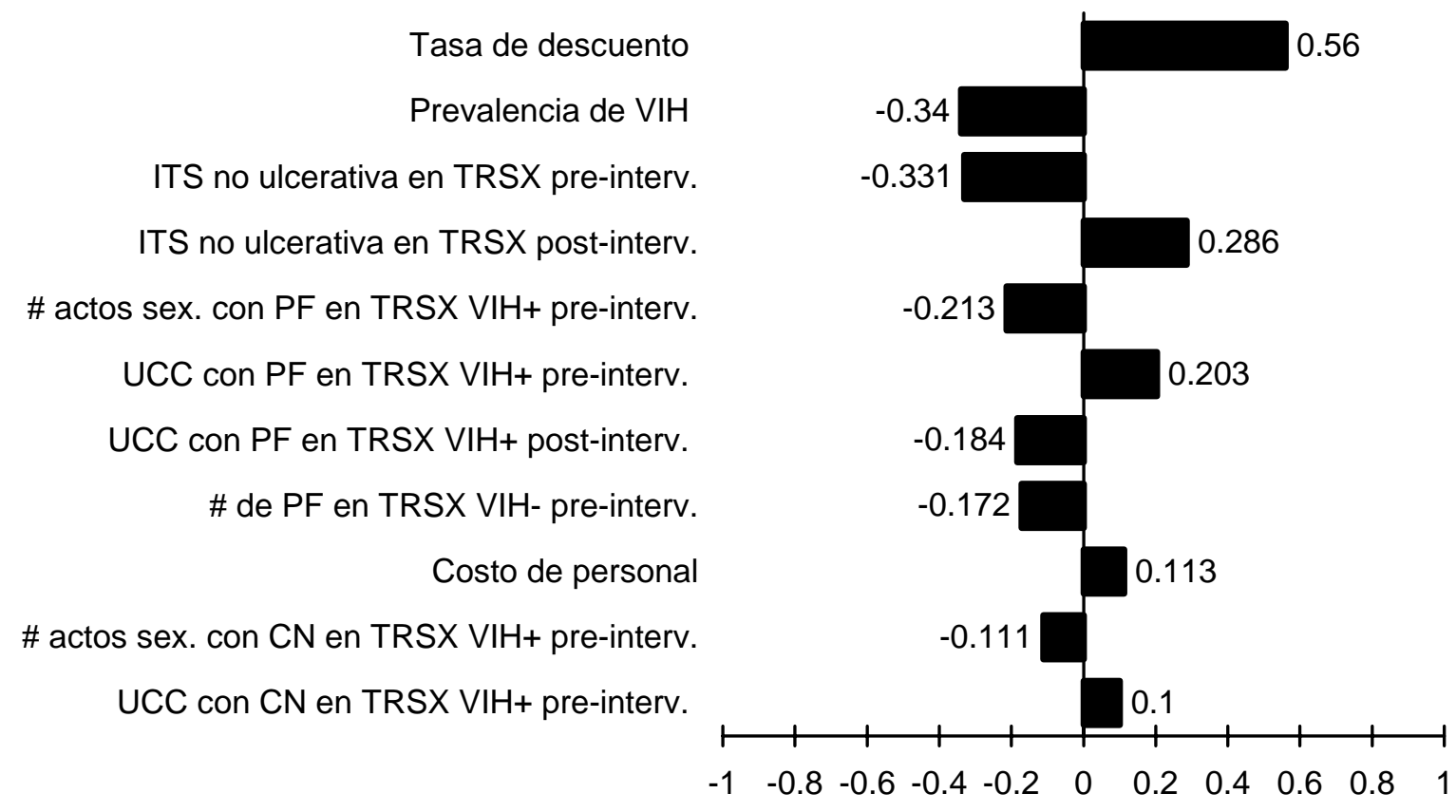

Coeficientes est. $b$ 


\section{Hgrizons}

\section{Discusión}

El interés en intervenciones ambientales-estructurales como estrategias efectivas para la prevención del VIH/ITS ha aumentado continuamente en los últimos años. Sin embargo, a pesar del aumento del interés y discurso sobre estos modelos, ha habido una disponibilidad muy limitada de datos que documenten la relación entre intervenciones ambientales-estructurales y reducciones específicas en el riesgo relacionado al VIH. Adicionalmente, la adaptabilidad de intervenciones ambientalesestructurales "exitosas" tampoco ha sido bien evaluada o documentada en la literatura (Parker et al. 2000). El estudio actual ayuda a llenar algunos vacíos de información demostrando la factibilidad y efectividad de una adaptación culturalmente apropiada del programa tailandés de 100 por ciento condón, ampliamente reconocido como una intervención ambiental-estructural efectiva en la prevención de VIH, a la industria de sexo comercial femenino en la República Dominicana.

Con los dos modelos de intervención, se documentaron cambios estadísticamente significativos de pre- a post-intervención para los tres resultados del estudio: uso consistente de condones, habilidad para rechazar verbalmente el sexo inseguro y prevalencia de ITS en ambas ciudades. Sin embargo, el tipo y nivel de estos cambios varió por la estrategia de intervención que fue utilizada. Por ejemplo, aumentos estadísticamente significativos en el uso consistente de condones fueron documentados con clientes nuevos en Santo Domingo, mientras aumentos significativos fueron encontrados con parejas fijas que pagan y que no pagan en le último mes solo en Puerto Plata. Estos cambios en el uso de condón con parejas fijas en el último mes son particularmente importantes dado que la encuesta pre-intervención documentó niveles de uso de condón más bajos en estas situaciones comparado con el uso de condón con clientes nuevos. Adicionalmente estos aumentos toman más importancia aún dado que la mayoría de interacciones de sexo en las trabajadoras sexuales que participaron en el estudio parecen estar ocurriendo con clientes repetidos o parejas fijas que pagan, basado en el número de citas con clientes reportado en la última semana y el número mediano total de parejas sexuales reportado por mes. Con los dos modelos de intervención, se documentaron reducciones significativas en ITS durante el transcurso de la intervención, aunque las reducciones documentadas fueron levemente más fuertes en Puerto Plata. Adicionalmente, solo en Puerto Plata se observó un aumento significativo de pre- a postintervención en la habilidad observada de verbalmente rechazar el sexo comercial inseguro en trabajadoras sexuales. La triangulación de los hallazgos del estudio indica que mientras que el modelo implementado puede haber tenido un impacto positivo en ambas ciudades en la reducción de la vulnerabilidad relacionada con el VIH en trabajadoras sexuales, el impacto de la intervención parece haber sido más amplio en Puerto Plata, donde se combinaron las estrategias de intervención de solidaridad y política gubernamental.

Una manera por la cual estos cambios fueron logrados en Puerto Plata parece estar relacionada con la capacidad del modelo combinado de solidaridad más política gubernamental de lograr más altos niveles de cumplimiento con los elementos de la intervención. Por ejemplo, en el análisis bivariable, exposición y cumplimiento con la intervención tienen asociaciones independientes y significativas con el uso consistente de condones con todas las parejas en el último mes, como la tiene la variable de ciudad. En el proceso de llegar al modelo multi-variable final, sin embargo, se encuentra que el efecto significativo de ciudad se pierde cuando el nivel de cumplimiento 
observado se agrega al modelo. Estos hallazgos sugieren que en lugares donde se puede lograr más altos niveles de cumplimiento, aumentos comparables en el uso de condones pueden ser posibles, independientemente de la ubicación geográfica. Sin embargo el estudio también encontró que solo fue en Puerto Plata donde se documentó niveles tan intensos de adopción y cumplimiento con los elementos claves de la intervención.

El análisis de costo-efectividad provee alumbramientos valientes a la factibilidad y ventaja relativa de la adopción de estos dos modelos competitivos de intervención. Un resultado claro de este análisis fue que era significativamente más costo-efectivo adoptar el modelo de intervención combinado que incluye la regulación y supervisión gubernamental. El análisis de costo-efectividad revela que el costo para implementar los dos modelos de intervención es comparable, pero con regulación reforzada con las autoridades de salud gubernamental existentes, la intervención es más de dos veces y media más costo-efectivo. Se encontró además que el modelo de intervención implementado en Puerto Plata que logró un nivel de US \$414 por AVAD salvado es una inversión bastante costo-efectivo en reducir la carga de VIH/SIDA en la República Dominicana.

Estos resultados fueron robustos por encima de un rango amplio de suposiciones. Aunque los datos sobre el costo de tratamiento son escasos, se puede suponer razonablemente que la intervención también ahorra costos en este ámbito. Por ejemplo, el nivel umbral de costo de tratamiento por vida para VIH/SIDA solo tendría que ser US \$9,843 para que los ahorros logrados con la intervención produjesen un ahorro solamente en los gastos médicos. El análisis de sensibilidad reveló que varios factores refuerzan el costo-efectividad de manera significativa, incluyendo la implementación de la intervención en áreas geográficas con tasas más altas de prevalencia de VIH en la población meta, especialmente donde el uso de condones es bajo con parejas fijas de trabajadoras sexuales. Ambos modelos de intervención probablemente son más costo-efectivos comparados con los otros modelos encontrados en la literatura en lugares parecidos.

Sobre la base de estos hallazgos, las personas involucradas en el estudio en la República Dominicana que trabajan con la planificación de programas y políticas están de acuerdo en que el modelo integrado de solidaridad y política en combinación con actividades continuas de educación entre pares y movilización comunitaria es un paquete de intervención apropiado, costo-efectivo y ético. Los resultados de este estudio, sin embargo, no contestan esta pregunta necesariamente para otros países. Cada país y comunidad tienen que examinar como las estrategias de solidaridad comunitaria y política gubernamental serían recibidos y funcionarían en sus propios contextos prácticos y políticos.

Aun así, los hallazgos de este estudio proveen evidencia que las intervenciones ambientalesestructurales que permiten y promueven tanto la eficacia individual como la colectiva para prevenir VIH son opciones importantes, efectivas y éticas que merecen consideración. Es más, la experiencia de la adaptación del modelo tailandés de 100 por ciento condones a la República Dominicana demuestra que iniciativas de política gubernamental para prevenir el VIH en el ámbito del trabajo sexual femenino no tienen que ser de arriba hacia abajo, sin participación comunitaria, ni violar los derechos humanos de trabajadoras sexuales (Loff et al. 2003), preocupaciones manifestadas por de miembros de las comunidades de activistas e investigadores. Al contrario, la política gubernamental puede apoyar a los miembros de la comunidad y los esfuerzos de las ONGs 


\section{Hgrizons}

de base comunitaria cuando todos estos actores participan en su construcción, implementación y evaluación.

El dialogo actual está enfocado en como expandir esta experiencia pilota exitosa de una manera que siga respetando a todos los miembros de las comunidades del trabajo sexual y que siga contribuyendo a frenar la epidemia de VIH de manera efectiva. Cualquier versión expandida tendrá que incluir un componente agregado que contiene actividades más intensas para involucrar a los clientes masculinos de trabajadoras sexuales. En un cuestionario breve con preguntas abiertas se les preguntó a las trabajadoras sexuales que participaron en el actual estudio y tuvieron pruebas positivas para ITS en la encuesta pre y post-intervención de cómo la intervención podría ayudarles en evitar la re-infección. Casi unánimemente las mujeres dijeron que los futuros esfuerzos de intervención tienen que aumentar y ampliar el trabajo con los clientes masculinos. Adicionalmente, la mayoría de las mujeres manifestaron la importancia de esfuerzos especiales para involucrar tanto sus parejas fijas que pagan como las que no pagan, por las cuales muchas sintieron haber sido infectadas. Actualmente, investigación formativa etnográfica está siendo realizada en la República Dominicana para explorar como involucrar a los clientes masculinos y movilizar su potencial para prevenir el VIH. 


\section{Limitaciones de la I nvestigación}

Una limitación del estudio es que el diseño de investigación pre-post test limita la capacidad de establecer asociaciones causales. Específicamente, con relación a los cambios en el riesgo de VIH de pre- a post-intervención, el estudio actual no puede establecer si los cambios que ocurrieron y las asociaciones encontradas entre altos niveles de exposición y cumplimiento con la intervención y el uso consistente de condones son resultados de la intervención. Sin embargo, para responder a las limitaciones de este diseño, se utilizó triangulación en la recolección de datos para variables del estudio y datos transversales mensuales para establecer tendencias temporales.

La coherencia interna en los tres resultados del estudio (el uso consistente de condones reportado, la habilidad observada de rechazar verbalmente el sexo inseguro, y los resultados de las pruebas de ITS) corroboran que mejoramientos significativos, particularmente en el caso de Puerto Plata, fueron logrados de pre- a post-intervención. Adicionalmente, los datos transversales mensuales de exposición y cumplimiento con la intervención ayudan a iluminar el mecanismo por el cual los modelos de intervención pueden haber tenido un efecto en la reducción de la vulnerabilidad de las trabajadoras sexuales a infectarse con VIH. Adjunto a la implementación de los modelos de solidaridad y política gubernamental, el diseño del estudio pudo haber sido fortalecido con la implementación de un modelo de intervención que consistía solamente en política gubernamental y regulación. Sin embargo, ninguna de las instituciones involucradas en el estudio creyó que un modelo solo con política gubernamental y regulación seria una estrategia ética de intervención, dado la importancia conceptual y el éxito programático de casi 15 años trabajando con esfuerzos de empoderamiento y movilización comunitaria con trabajadoras sexuales en el contexto dominicano. 


\section{Hgrizons}

\section{Conclusiones}

Los dos modelos adaptados de 100 por ciento condones implementados como parte de este estudio en la República Dominicana tuvieron un impacto positivo en los resultados específicos relacionados al VIH. Sin embargo, los hallazgos del estudio sugirieron que un modelo integrado incluyendo una estrategia de solidaridad basada en la comunidad y una estrategia de política gubernamental y regulación enfocado en la prevención del VIH y el uso de condones logró mayores aumentos en el comportamiento relacionado a la prevención del VIH además de mayores reducciones en ITS en trabajadoras sexuales. Este modelo también fue más costo-efectivo. Sin embargo, para que los logros programáticos sean sostenibles, las intervenciones ambientalesestructurales tienen que involucrar a los miembros de la comunidad en la conceptualizacion, implementación y evaluación de iniciativas basadas en la política gubernamental para asegurar no solo que sean efectivas sino que también sean aceptables y apropiadas para reducir el riesgo relacionado al VIH en el contexto del trabajo sexual femenino. 


\section{Referencias}

Aggleton, Peter. 1997. "Behavior change communication strategies," AIDS Education and Prevention 9(2): 111-123.

AIDSCAP/FHI." 1993. Technical Assessment of AIDS in the Dominican Republic: A Report to the U.S. Agency for International Development.” Santo Domingo: AIDSCAP/FHI.

Aklilu, Mathias et al. 2001. "Factors associated with HIV-1 infection among sex workers of Addis Ababa, Ethiopia," AIDS 15: 87-96.

Asamoah-Adu, Comfort et al. 2001. "HIV Infection Among Sex Workers in Accra: Need to Target New Recruits Entering the Trade," Journal of Acquired Immune Deficiency Syndromes 28: 358366.

Auerbach, Judith D. and Thomas J. Coates. 2000. "HIV prevention research: accomplishments and challenges for the third decade of AIDS," American Journal of Public Health 90(7): 1029-1032.

Bhave, G. et al. 1995. "Impact of an intervention on HIV, sexually transmitted diseases, and condom use among sex workers in Bombay, India," AIDS 9 (Suppl 1): S21-30.

Busza, Joanne and B.T. Schunter. 2001. "From competition to community: participatory learning and action among young, debt-bonded Vietnamese sex workers in Cambodia," Reproductive Health Matters 9(17): 72-81.

Campbell, Catherine. 2000. "Selling sex in the time of AIDS: the psycho-social context of condom use by sex workers on a Southern African mine," Social Science \& Medicine 50(4): 479-94.

Campbell, Catherine and Zodwa Mzaidume. 2001. "Grassroots participation, peer education, and HIV prevention by sex workers in South Africa," American Journal of Public Health 91(12): 1978-1986.

Celentano, David D. et al. 1998. "Decreasing incidence of HIV and sexually transmitted diseases in young Thai men: evidence for success of the HIV/AIDS control and prevention program," AIDS 12(5): 29-36.

CESDEM (Centro de Estudios Sociales y Demograficos). 1996. "Informe de Resultados. Encuesta sobre Conocimientos, Creencias, Actitudes y Prácticas acerca del VIH/SIDA en Trabajadoras Sexuales y Hombres Involucrados en La Industria del Sexo en Santo Domingo" [Final Report. Survey of knowledge, beliefs, attitudes and practices in relation to HIV/AIDS among Female Sex Workers and Men Involved in the sex industry in the Dominican Republic], Santo Domingo: CESDEM. 


\section{Hgrizons}

1999. "Informe de Resultados. Encuesta sobre Conocimientos, Creencias, Actitudes y Prácticas acerca del VIH/SIDA en Trabajadoras Sexuales y Hombres Involucrados en La Industria del Sexo de la República Dominicana" [Final Report. Survey of knowledge, beliefs, attitudes and practices in relation to HIV/AIDS among Female Sex Workers and Men Involved in the sex industry in the Dominican Republic], Santo Domingo: CESDEM.

2003. Encuesta Demografica y de Salud 2002: Informe Preliminar sobre VIH/SIDA. [Demographic Health Survey 2002: preliminary results regarding HIV/AIDS]. Santo Domingo: CESDEM.

COIN (Centro de Orientacion e Investigacion Integral). 1998. "Census of commercial sex establishments," Santo Domingo: COIN.

- 2000. Personal communication. Santo Domingo.

DIGECITSS (Dirección General del Control de las Enfermedades de Transmisión Sexual y SIDA. 2000. "Vigilancia Centinela de la Infección VIH. Informe de Monitoreo 10 mo. año" [Sentinel Surveillance of HIV Infection. Monitoring report from 10th year], Santo Domingo: SESPAS.

Evans, Catherine. 1999. "An international review of the rationale, role and evaluation of community development approaches in interventions to reduce HIV transmission in sex work," Horizons Program Report. New Delhi: Population Council.

Ford, Kathleen et al. 1996. "Behavioral interventions for reduction of sexually transmitted disease/HIV transmission among female commercial sex workers and clients in Bali, Indonesia," AIDS 10(2): 213-222.

Futures Group. 2002. Documenting the experience of sex workers. Report to the Future's Group Policy Project from David Lowe Consulting/Asia. Washington, D.C.: Futures Group.

Ghys, Peter D. et al. 2001. "Effect of interventions to control sexually transmitted disease on the incidence of HIV infection in female sex workers," AIDS 15: 1421-1431.

Gray, Jennifer A. et al. 1997. "HIV-1 infection among female commercial sex workers in rural Thailand," AIDS 11(1): 89-94.

Gysels, Marjolein, Robert Pool, and Betty Nnalusiba. 2002. "Women who sell sex in a Ugandan trading town: life histories, survival strategies and risk," Social Science and Medicine 54(2): 179192.

Hanenberg, Robert S. et al. 1994. "Impact of Thailand's HIV-control programme as indicated by the decline of sexually transmitted diseases," Lancet 344(8917): 243-245. 
Hendriks, J.C. et al. 1996. "Estimation of progression of HIV infection among intravenous drug users using a death-included staged Markov model," paper presented at the $11^{\text {th }}$ International Conference on AIDS, Vancouver, Canada (Abstract no. Th.C.223).

Jana, Smarajit et al. 1998. "STD/HIV intervention with sex workers in West Bengal, India," AIDS 12 (Suppl B): S101-108.

Kalipeni, Ezekiel. 2000. "Health and disease in southern Africa: a comparative and vulnerability perspective," Social Science \& Medicine 50(7-8): 965-983.

Kerrigan, Deanna. 2000. "Individual, Relational, and Environmental-Structural Determinants of Consistent Condom Use Among Female Sex Workers and their Regular Partners in the Dominican Republic," Doctoral Dissertation. Baltimore: Johns Hopkins School of Public Health.

Kerrigan, Deanna et al. 1997. "Formative Research for a 100\% Condom Program in the Dominican Republic," Santo Domingo: AIDSCAP/FHI/USAID.

Kerrigan, Deanna et al. 2001. "Adapting the Thai 100\% condom programme: developing a culturally appropriate model for the Dominican Republic," Culture, Health and Sexuality 3(2): 221-240.

Kilmarx, Peter H. et al. 1998. "HIV-1 seroconversion in a prospective study of female sex workers in northern Thailand: continued high incidence among brothel-based women," AIDS 12(14): 18891898.

Laga, Marie et al. 1994. "Condom promotion, sexually transmitted diseases treatment, and declining incidence of HIV-1 infection in female Zairian sex workers," Lancet 344(8917): 246-248.

Leonard, Lori, et al. 2001. "HIV prevention among male clients of female sex workers in Kaolack, Senegal: results of a peer education program," AIDS Education and Prevention 12(1):21-37.

Loff, Bebe, Cheryl Overs, and Paulo Longo. 2003. "Can health programmes lead to the mistreatment of sex workers?" Lancet 361(9373): 1982.

Mason, C.J. et al. 1995. "Declining prevalence of HIV-1 infection in young Thai men," AIDS 9(9): 1061-1065.

Mehendale, Sanjay M. et al. 1995. "Incidence and predictors of human immunodeficiency virus type 1 seroconversion in patients attending sexually transmitted disease clinics in India," Journal of Infectious Diseases 172(6): 1486-1491.

Merson, Michael H., J.M. Dayton, and Kevin O'Reilly. 2000. "Effectiveness of HIV prevention interventions in developing countries," AIDS 14(Suppl 2): S68-84. 


\section{Hgrizons}

Moreno, Luis and Deanne Kerrigan. 2000. "HIV Prevention strategies among female sex workers in the Dominican Republic," Research for Sex Work 3: 8-10.

Morisky, Donald et al. 2002. The impact of the work environment on condom use among female bar workers in the Philippines. Health Education and Behavior. Aug;29(4):461-72.

Morisky, Donald E. et al. 1998. "The effects of establishment practices, knowledge and attitudes on condom use among Filipina sex workers," AIDS Care 10(2): 213-220.

Murray, C. J. L. and A.D. Lopez. 1996. "The global burden of disease: a comprehensive assessment of mortality and disability from diseases, injuries, and risk factors in 1990 and projected to 2020," Cambridge: Harvard University Press.

Nelson, Kenrad E. et al. 1996. "Changes in sexual behavior and a decline in HIV infection among young men in Thailand," New England Journal of Medicine 335(5): 297-303.

Nelson, Kenrad E. et al. 2002. "HIV infection in young men in northern Thailand, 1991-1998: Increasing role of injection drug use," Journal of Acquired Immune Deficiency Syndrome 29(1): $62-68$.

Ngugi, Elizabeth N. et al. 1996. "Focused peer-mediated educational programs among female sex workers to reduce sexually transmitted disease and human immunodeficiency virus transmission in Kenya and Zimbabwe," Journal of Infectious Diseases 174 (Suppl 2): S240-S247.

O'Reilly, Kevin R. and Peter Piot. 1996. "International perspectives on individual and community approaches to the prevention of sexually transmitted disease and human immunodeficiency virus infection," Journal of Infectious Diseases 174 (Suppl 2): S214-222.

Pareja, Reynaldo and Santo Rosario. 1992. "Sexo, Trabajo y Sociedad" [Sex, Work, and Society,] Santo Domingo: La Unión.

Parker, Richard et al. 2002. "HIV/AIDS-related stigma and discrimination: A conceptual framework and an agenda for action," Horizons report. Washington, D.C.: Population Council.

Parker, Richard F., D. Easton, and C.H. Klein. 2000. "Structural barriers and facilitators in HIV prevention: a review of international research," AIDS 14 (Suppl 1): S22-32.

Quan, Vu Minh et al. 2000. "HIV in Vietnam: the evolving epidemic and the prevention response, 1996 through 1999," Journal of Acquired Immune Deficiency Syndrome 25(4): 360-369.

Rehle, T. et al. 1998. "AVERT: A User Friendly Model to Estimate the Impact of HIV/Sexually Transmitted Disease Prevention Interventions on HIV Transmission," AIDS 12: S27-S35.

Rojanapithayakorn, Wiwat and Robert Hanenberg. 1996. "The 100\% condom program in Thailand," AIDS 10(1): 1-7. 
Rosario, Santo et al. 1994. "La Industria de Sexo por Dentro," Santo Domingo: COIN.

Sakondhavat, Chuanchom et al. 1997. "Promoting condom-only brothels through solidarity and support for brothel managers," International Journal of STD and AIDS 8: 40-43.

Sumartojo, E. (ed.). 2000. "Structural factors and HIV prevention," AIDS 14 (Suppl 1): S3-S10.

Sweat, Michael and Julie Denison. 1995. "Reducing HIV incidence in developing countries with structural and environmental interventions," AIDS 9 (Suppl A): S251-S257.

Tawil, Oussama, A.Verster, and Kevin R. O'Reilly. 1995. "Enabling approaches for HIV/AIDS prevention: can we modify the environment and minimize the risk?" AIDS 9: 1299-1306.

Tiglao, Teodoro V. et al. 1996. "A community P.A.R. Approach to HIV/AIDS prevention among sex workers," Promotion and Education 3(4): 25-28.

—. 2002. "Sex work and HIV/AIDS. Technical Update," UNAIDS Best Practice Collection. Geneva: UNAIDS.

UNFPA (United Nations Population Division). 2001. "World Population Prospects: The 2000 Revision Highlights," Population Division of the Department of Economic \& Social Affairs of the United Nations Secretariat.

Visrutaratna, S. 1995. “'Superstar' and 'model brothel': developing and evaluating a condom promotion program for sex establishments in Chiang Mai, Thailand," AIDS 9(Suppl 1): S69-75.

Waldo, Craig R. and Thomas J. Coates. 2000. "Multiple levels of analysis and intervention in HIV prevention science: exemplars and directions for new research," AIDS 14(Suppl 2): S18-26.

Weinstein, M. et al. 1989. "Cost-effectiveness analysis of AIDS prevention programs: concepts, complications, and illustrations," Washington, D.C.: National Academy Press.

World Bank. 1993. "World Development Report: Investing in Health,” New York: Oxford University Press. 


\section{Hqrizons}

Horizons es un programa de investigación operativa global cuyos objetivos son:

- Identificar y evaluar estrategias potenciales para mejorar programas de prevención, cuidado y apoyo del SIDA y la entrega de servicios.

- Difundir mejores prácticas y promover la utilización de los resultados con el objetivo de replicar intervenciones exitosas.

\section{(2) Population Council}

\section{El Population Council implementa} Horizons en colaboración con

- International Center for Research on Women (ICRW)

- International HIV/AIDS Alliance

- Program for Appropriate Technology in Health (PATH)

- Tulane University

- Family Health International (FHI)

- Johns Hopkins University

Para más información acerca del estudio, contacte:

Horizons Program, Communications Unit 4301 Connecticut Avenue, NW Suite 280 Washington, DC 20008 USA

Tel: 202-237-9400

Fax: 202-237-8410

Email: horizons@pcdc.org

www.popcouncil.org/horizons 